\title{
Räume unter Strom
}

\section{Eine diskurstheoretische Analyse zu Aushandlungsprozessen im Zuge des Stromnetzausbaus}

\author{
Florian Weber ${ }^{1}$ Olaf Kühne ${ }^{1}$
}

Eingegangen: 7. Dezember 2015 / Angenommen: 29. Juni 2016 / Online publiziert: 19. Juli 2016

(C) Springer-Verlag Berlin Heidelberg 2016

Zusammenfassung Die Energiewende verändert in Deutschland mit dem Ausstieg aus der Kernkraft und dem Ausbau erneuerbarer Energien in weitreichender Weise bisherige Strukturen der Energieversorgung und wirkt sich dabei räumlich stark aus. Einen Aspekt bilden hierbei Veränderungen im bestehenden Stromnetz. Vorhandene Leitungstrassen sollen ertüchtigt, andere umfänglich neu gebaut werden, was Widerstände und Konflikte mit sich bringt. Der Artikel untersucht vor diesem Hintergrund aus diskurstheoretischer Perspektive, wie der Stromnetzausbau und mögliche Widerstände verhandelt werden und welche Argumentationsmuster dabei vorherrschend sind. Die durchgeführten Analysen fußen auf einem Methodenmix aus quantitativ orientierten und qualitativen Analysebestandteilen, um sowohl zentrale Bezugnahmen auszudifferenzieren als auch Einzelaspekte detaillierter zu betrachten. Zusammenfassend bilden die Bedarfsfrage des Stromnetzausbaus, Beteiligung, die eingesetzte Technik, Gesundheit, Wirtschaft sowie Natur und Landschaft zentrale Konfliktfelder, die innerhalb des Stromnetzausbaus ausgehandelt werden und sich in eher kognitive, emotionale und ästhetische Bewertungsmuster einreihen.

Schlüsselwörter Energiewende - Erneuerbare Energien · Stromnetzausbau $\cdot$ Diskurstheorie $\cdot$ Macht

Dr. Florian Weber

florian.weber@hswt.de

Prof. Dr. Dr. Olaf Kühne

olaf.kuehne@hswt.de

1 Landschaftsarchitektur, Hochschule Weihenstephan-Triesdorf, Am Hofgarten 6, 85354 Freising, Deutschland

\section{Spaces and Power Lines}

A discourse-theoretical analysis of negotiation processes in the course of the national power grid extension

Abstract With its phase-out of nuclear power generation and the expansion of renewably sourced energy, the German energy transition ("Energiewende") has radically changed existing power supply structures, with wide geographical repercussions. One aspect of this is changes within the being national power grid. Existing networks are to be upgraded and new long-distance power-lines built. This creates resistance and conflict. Against this background, our article investigates from a discourse theory perspective how power grid extensions and possible opposition are communicatively constructed, and what argumentation patterns dominate the discussion. The analyses undertaken here are based on a methodological mix of quantitativeoriented and qualitative elements that facilitates both the differentiation of central issues and detailed examination of individual aspects. In sum, the underlying need for grid extension and the technology involved on the one hand, and issues of health, local economies, natural environment, landscape, and participation in the decision-making process on the other hand, form central fields of conflict to be negotiated at cognitive, emotional and aesthetic levels of discourse.

Keywords Energy transition - Renewable energies · Power grid extension · Discourse theory $\cdot$ Power

\section{Einleitung: Konfliktfeld Stromnetzausbau}

Vor dem Hintergrund der Reaktorkatastrophe in Fukushima im März 2011 wurden der Ausstieg aus der Kernkraft 
bis 2022 und der weitere Ausbau erneuerbarer Energieträger Kernstücke politischen Agierens (vgl. unter anderem Bundesregierung 2015). Dieser Ausbau wird in Deutschland generell weithin als notwendig, in großen Teilen sogar als wünschenswert betrachtet. Nach einer repräsentativen Umfrage des Meinungsforschungsinstituts TNS Emnid vom August 2015 bewerteten $93 \%$ der Befragten den weiteren Ausbau als „wichtig“ bis ,außerordentlich wichtig“ (Agentur für Erneuerbare Energien 2015: o. S.). Der grundsätzlichen Zustimmung zum Trotz entzünden sich Konflikte auf lokaler Ebene an konkreten Planungen von Windkraft-, Photovoltaik- oder Biomasseanlagen (vgl. Leibenath/Otto 2013). Zudem ergeben sich Konfliktfelder im Zuge des geplanten Stromnetzausbaus - und dies nicht allein punkthaft an einzelnen Orten, sondern vielmehr linienhaft und vernetzt: Landkreise, Kommunen und Bürgerinitiativen schlieBen sich zu Bündnissen zusammen, tauschen sich aus und streben Anpassungen von Planungen an oder haben zum Ziel, ganze Trassen zu verhindern (z.B. Feneberg 2014; Landkreis Hameln-Pyrmont 2014).

Der Ausbau bestehender Stromnetze ist allerdings nicht erst ein Phänomen der Post-Fukushima-Ära. Im Jahr 2009 wurden im Energieleitungsausbaugesetz (EnLAG) Vorhaben mit fast $2000 \mathrm{~km}$ Länge festgelegt (Riegel/Brandt 2015: 10), wobei darin enthaltene bereits vor Inkrafttreten des Gesetzes initiiert wurden und sich an einem Streckenabschnitt beispielsweise bereits 2004 eine Bürgerinitiative gründete (Neukirch 2014: 23). Die Perspektive, dass bis 2022 alle deutschen Kernkraftwerke vom Netz gehen sollen, führte jedoch zur dezidierten Benennung weiterer Vorhaben mit ,vordringlichem Bedarf“: Im Bundesbedarfsplangesetz (BBPIG), das im Juli 2013 in Kraft trat, wird ein „Bedarf für rund 5000 Kilometer Netzausbau- und Netzverstärkungsmaßnahmen“ konstatiert (Riegel/Brandt 2015: 10) - begründet mit dem Verweis darauf, Energie aus dem windreichen Norden müsse in den verbrauchsstarken Süden transportiert werden. Für Windkraftanlagen günstige windhöffige Standorte „,befinden sich [gerade] in den strukturschwachen und dünn besiedelten Regionen Norddeutschlands bzw. in der Nord- und Ostsee“ (Neukirch 2014: 5). $\mathrm{Zu}$ den Vorhaben gehören auch der "SuedLink“ und die „Gleichstrompassage Süd-Ost“, die in Gleichstromübertragungstechnik errichtet werden sollen und zwischenzeitlich $\mathrm{zu}$ besonders massivem Widerstand, gerade im Freistaat Bayern, führten. Während, vereinfachend dargestellt, die Energieleitungsausbaugesetz-Vorhaben in Länderzuständigkeit fallen, werden für länderübergreifende Bundesbedarfsplangesetz-Vorhaben die Bundesfachplanungen und die Planfeststellungsverfahren durch die Bundesnetzagentur durchgeführt ${ }^{1}$, womit sich Planungszuständigkeiten

\footnotetext{
${ }_{1}^{1} \mathrm{http} / /$ www.netzausbau.de/wissenswertes/recht/nabeg/de.html (13.06.2016).
}

verschieben, ebenso wie sich Veränderungen im Hinblick auf Beteiligungsverfahren ergeben (Schmidt 2015: 17). Die Übertragungsnetzbetreiber 50 Hertz, TenneT, Amprion und TransnetBW erstellen Netzentwicklungspläne mit als erforderlich angesehenen Ausbauvorhaben, die durch die Bundesnetzagentur geprüft werden. Auch hieran entzünden sich Konflikte, unter anderem mit dem Verweis auf unzureichende Transparenz (Stegert/Klagge 2015: 172).

Der Prozess des Stromnetzausbaus wird zu einem Feld unterschiedlicher Aushandlungsprozesse und Widerstände. In bisherigen Veröffentlichungen wurden besonders fallstudienbezogen Akzeptanzfragen sowie Medienberichterstattung und Expertenpositionen fokussiert, die Entwicklungen bis Jahresende 2012 (vgl. Schweizer-Ries 2010; Zimmer/Kloke/Gaedtke 2012; Hübner/Hahn 2013) bzw. Herbst 2013 (Neukirch 2014) abdecken. Darüber hinaus wurden Beteiligungsverfahren zu Projekten im Kontext des Energieleitungsausbaugesetzes (Koch/Odparlik/Köppel 2014) sowie Stellungnahmen zu Konsultationsverfahren zum Netzentwicklungsplan als Grundlage für Überlegungen zur Bürgerbeteiligung (Stegert/Klagge 2015) analysiert. Der deutlich angewachsene Widerstand, der im Juli 2015 in der politischen Entscheidung, Erdverkabelungen zu favorisieren, mündete (vgl. Abb. 4), wurde für die Gleichstrompassage Süd-Ost von Neukirch (2015) aufgegriffen, aber noch nicht mit Fallstudien beleuchtet. Auch standen bis dato Verfestigungsprozesse bestimmter Deutungsmuster - Befürwortung und Gegnerschaft des Netzausbaus multiperspektivisch weniger im Mittelpunkt.

Im Rahmen eines Forschungsvorhabens im Auftrag des Bundesamtes für Strahlenschutz wurden von November 2014 bis Oktober 2015 zentrale Argumentationsmuster und Konfliktlinien in den Mittelpunkt gerückt. Ausgehend von einer diskurstheoretischen Perspektive wurden aktuelle Aushandlungsprozesse - innerhalb medialer Darstellungen sowie experten- und fallstudienbezogen - ausdifferenziert. Es rückt damit eine Forschungsperspektive in den Mittelpunkt, die die Herstellung "sozialer Wirklichkeit" und die Verfestigung bestimmter Bedeutungen fokussiert. Der Beitrag geht vor diesem Hintergrund der Frage nach, welche zentralen Diskursstränge und darin enthaltene Hauptargumentationsmuster, insbesondere der Kritik, sich im Zuge der Aushandlungsprozesse um den Stromnetzausbau nachzeichnen lassen. Welche Positionen sind machtvoll hegemonial - verankert, welche werden dagegen eher in den Hintergrund gedrängt? Welche Bruchlinien lassen sich dabei gegebenenfalls auch herausarbeiten?

Zunächst werden im Folgenden die diskurstheoretische Grundperspektive und das methodische Vorgehen sowie Analysebestandteile erläutert (Abschn. 2). Im Anschluss werden zentrale Argumentationsmuster der Befürwortung und der Gegnerschaft zum Stromnetzausbau unter Einbeziehung fallstudienbezogener Spezifika dargestellt (Ab- 
schn. 3). Vor dem Hintergrund der Ergebnisse werden abschließend Zusammenhänge und mögliche Folgen für zukünftige Entwicklungen skizziert (Abschn. 4).

\section{Diskurstheoretische Forschungsperspektive, methodisches Vorgehen und Analysebestandteile}

\subsection{Die diskurstheoretische Grundperspektive}

Planungen um den Stromnetzausbau zeigen mit einem Blick auf die Entwicklungen der letzten Jahre, dass es hierbei immer wieder zu verschiedenen Veränderungen gekommen ist, wie beispielsweise die Definition von Vorhaben über das Bundesbedarfsplangesetz neben den bereits bestehenden Vorhaben aus dem Energieleitungsausbaugesetz, die Übernahme/Nicht-Übernahme von Vorschlägen der Übertragungsnetzbetreiber in die Netzentwicklungspläne oder im Hinblick auf Freileitungen gegenüber Erdverkabelungen, die gerade beim SuedLink oder der Gleichstrompassage Süd-Ost zunächst nicht vorgesehen waren. Gleichzeitig lässt sich beobachten, dass bestimmte Positionen, wie die Legitimation des Netzausbaus über den Ausstieg aus der Kernkraft und den weiteren Ausbau erneuerbarer Energien gerade in Norddeutschland recht fest verankert und unumstößlich scheinen. Veränderungen einerseits und verfestigte Setzungen andererseits lassen sich als zwei Seiten einer Medaille verstehen, die gerade aus einer diskurstheoretischen Forschungsperspektive in ihrem Wechselspiel beleuchtet werden können.

Ausgangspunkt bildet in strukturalistischer und poststrukturalistischer Tradition der Zugang zur ,sozialen Wirklichkeit" über Sprache, durch die Bedeutungen relational über Differenzbeziehungen verankert werden (vgl Abb. 1, vgl. ausführlich Glasze 2013). Sprache kann als zentraler Mechanismus bei der Vermittlung einer „objektivierten“ sozialen Welt gedeutet werden, die Wahrnehmungen strukturiert und spezifische Wirklichkeiten herstellt (Torfing 1999: 87; Phillips/Jørgensen 2002: 8 f.; Gailing/Leibenath 2015: 126 f.). Durch regelmäßige Wiederholung werden bestimmte Deutungen verfestigt - sie erscheinen in der Folge im Alltag als gegeben und „normal“. Entsprechend werden sie nicht hinterfragt. Dadurch wird Bedeutung temporär fixiert - von Laclau und Mouffe (1985: 112) als Diskurs gefasst (vgl. auch erläuternd Glasze 2013: 75 f.). Diskurse umfassen „Sprache, Subjekte, nicht-sprachliche Praktiken und Objekte", die miteinander verbunden sind also die Gesamtheit des Diskurses bilden (Leibenath 2014: 125). Beispielhaft vereinfachend lässt sich dies am Diskurs um den Klimaschutz verdeutlichen, der unter anderem mit Kohlenstoffdioxid-Reduktion und Ressourcenschonung verknüpft wird und bei dem eine Abgrenzung von Treibhausgasemissionen, Rodung des tropischen Regenwaldes und Ressourcenverschwendung erfolgt. Gleichzeitig sind immer Veränderungen möglich - es bestehen regelmäßig unterschiedliche Diskursstränge, die miteinander konkurrieren und bei denen auch bisher eher unterdrückte Deutungen zentral werden könn(t)en. Laclau und Mouffe (1985) betonen damit die Unmöglichkeit endgültig fixierter Strukturen. Auch in vermeintlich stabilen Verhältnissen können Veränderungen auftreten (Laclau 1994: 1 f.; Weber 2013: 50). Bedeutungsveränderungen sind vom Grundsatz her immer möglich. Aufgrund dieser Veränderlichkeit sind Deutungshoheiten temporär, wodurch diese einem ständigen Prozess der Verteidigung, Erosion, Umdefinition und Neuerrichtung, kurz von Macht, unterliegen. Dies trifft beispielsweise auf die Bewertung der in Deutschland in den 1960er-Jahren von vielen als zukunftsweisend erachteten und heute eher mit Risiken verknüpften Kernkraftwerken zu (Gleitsmann 2011: 20, 23).

Entscheidend ist vor diesem Hintergrund, welche Diskursstränge - konkret innerhalb des Stromnetzausbaus so unhinterfragt werden, dass ihre Veränderungsmöglichkeit in Vergessenheit gerät und sie unverrückbar scheinen. Solche Diskurse werden von Laclau und Mouffe als besonders machtvolle und erfolgreiche - hegemoniale - Diskurse bezeichnet. Verschiedene Momente reihen sich hierbei in Äquivalenzketten um einen zentralen Knotenpunkt herum aneinander, womit der Diskurs repräsentiert wird (Phillips/Jørgensen 2002: 26 f.). Gleichzeitig ergeben sich Abgrenzungen von einem Außen, also dem, was der Diskurs nicht ist (vgl. Abb. 1). Dieses Außen wird so identitätsstiftend - als konstitutives, antagonistisches Außen für den Diskurs zu betrachten (Laclau 1993; Thiem/Weber 2011: 175 f.; Weber 2015: 102). Alternative Deutungen werden dadurch - zumindest zeitweise - unterdrückt (Laclau 1993; Glasze/Mattissek 2009a: 162).

Marginalisierte Diskursstränge lassen sich als Subdiskurse begreifen, die im Schatten hegemonialer Diskurse stehen, aber, wie erläutert wurde, vom Prinzip her auch hegemonial werden könnten (Weber 2013: 63, 69). Machtfragen erhalten so zentrale Relevanz, also welche Diskurse sich im Vergleich zu anderen in Aushandlungsprozessen durchsetzen und verfestigen, womit gleichzeitig das Sagbare definiert und diskursiv verfestigt wird (vgl. auch Glasze 2013; Weber 2013).

\subsection{Methodisches Vorgehen: Systematisierung von Sprecherpositionen und Analyse narrativer Muster}

Laclau und Mouffe haben in ihren Arbeiten primär ein „Interesse an politiktheoretischen Zusammenhängen und philosophischen Beweisen“ (Leibenath/Otto 2012: 123) verfolgt und nur wenige Hinweise zu einer empirischen Operationalisierung gegeben (Glasze 2013: 97). Um den theoretischen Implikationen der Theorie Rechnung zu tragen, sind 
Abb. 1 Diskursverständnis Knotenpunkt mit angereihten Momenten, Grenzziehung sowie Außen des Diskurses. (Glasze 2013: 83, verändert)

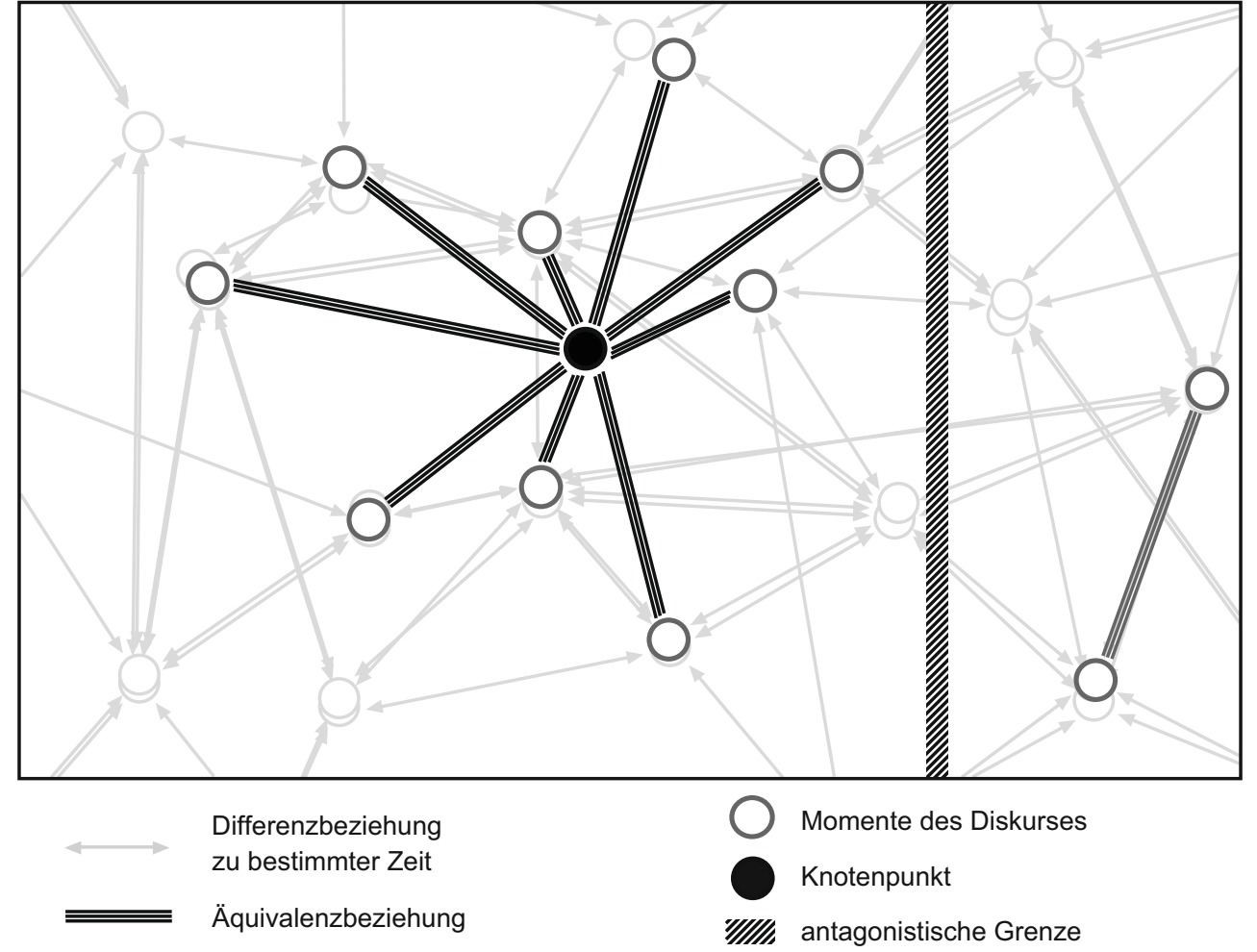

angepasste Methoden erforderlich (vgl. Glasze/Mattissek 2009b). Wie in Abschn. 2.1 ausgeführt wurde, besteht der Ansatzpunkt der Diskurstheorie in der Deutung von Diskursen als temporäre Fixierung von Bedeutung, die gerade dadurch hergestellt wird, dass vergleichbare Muster regelmäßig (re)produziert werden. Alternative Deutungsmöglichkeiten rücken so ins Abseits. Methodisch ist also darauf zu achten, Regelmäßigkeiten herauszuarbeiten, mit denen spezifische Deutungsmuster verfestigt werden.

Um Positionen und Hegemonien im Diskurs abzubilden, wurden innerhalb der Analysebestandteile zitierte Sprecher und deren Sprecherpositionen ausdifferenziert und quantifiziert. Unter Sprecherpositionen werden ,institutionell stabilisierte Positionen“ (Glasze 2007) verstanden, also Positionen, die Teil von Diskursen sind und von Sprechern (also Subjekten) (re)produziert werden. Hierbei wurden die Untersuchungsmaterialien Google-Treffer, SZ- und FocusArtikel, die Artikel der regionalen und lokalen Zeitungen zu den Fallstudien sowie die Internet-/Facebook-Seiten der Bürgerinitiativen (vgl. Abschn. 2.3) durch ein Mitglied des Projektteams gelesen, durch weitere Mitglieder gegengeprüft und ausgewertet. Ein automatisiertes Vorgehen mit computergestützter Software wie bei lexikometrischen Verfahren in Bezug auf hegemonial verankerte Wörter und Wortfolgen (vgl. z. B. Weber 2015) ist nicht möglich, da sich dieser methodische Zugang an der Grenze zu qualitativen Verfahren bewegt, wenn auch ein quantifizierendes Ergebnis angestrebt wird.
Um „überindividuelle, ,hegemoniale“ Muster des Sprechens über einzelne Themen“ (Mattissek 2008: 115) nachzuzeichnen, wurde die Analyse narrativer Muster herangezogen (Glasze/Husseini/Mose 2009). Sie untersucht, „wie Bedeutungen konstituiert werden, indem sprachliche Elemente in bestimmte Muster eingebunden werden und gewisse Regelmäßigkeiten offenbaren“ (Weber 2013: 67). Der Fokus lässt sich damit gerade auf Äquivalenzbeziehungen und Gegensätze richten (Somers 1994: 616), um so Momente des Diskurses in Beziehung zu setzen, zentrale Knotenpunkte zu identifizieren und nachzuzeichnen, wovon jeweils Abgrenzungen - dem Außen des Diskurses - erfolgen. Regelmäßig werden spezifische Narrationen genutzt, die durch ihre Wiederholung verfestigt werden und zur Bedeutungsfixierung beitragen (Glasze/Husseini/Mose 2009: 293 f.). Zielsetzung der Analyse ist, diese Regelmäßigkeiten herauszuarbeiten. So können zentrale Momente und Knotenpunkte sowie Grenzziehungen aufgefächert werden, die spezifische Deutungsmuster im Zuge der Aushandlungsprozesse um den Stromnetzausbau (re)produzieren.

\section{3 Übersicht über die Bestandteile der Analysen}

Innerhalb der durchgeführten Analysen wurden mediale Repräsentationen, Einschätzungen von Interviewpartnern mit Bundes- und Länderbezug sowie lokale Fallstudien berücksichtigt, um einen multiperspektivischen Blick zu ermöglichen (vgl. Tab. 1).

Dem Internet kommt als Informations- sowie Willensbildungs- und Partizipationsplattform wachsende Bedeutung 
Tab. 1 Übersicht über die Analysebestandteile

\begin{tabular}{|c|c|c|c|c|}
\hline Ebene & & Medium/Interviewpartner & Zeitpunkt/Zeitraum & Kürzel \\
\hline \multirow[t]{15}{*}{$\overline{\text { Bundesweit }}$} & & $\begin{array}{l}50 \text { erste Google-Treffer zum Schlagwort „Stromnetzausbau“ } \\
\text { http://www.google.de }\end{array}$ & 04.03 .2015 & $\begin{array}{l}\text { GT01 bis } \\
\text { GT50 }\end{array}$ \\
\hline & & $\begin{array}{l}\text { Tageszeitung Süddeutsche Zeitung (SZ) } \\
\text { http://www.sueddeutsche.de }\end{array}$ & 17.05.2010-23.04.2015 & SZ \\
\hline & & $\begin{array}{l}\text { Wochenmagazin Focus } \\
\text { http://www.focus.de }\end{array}$ & 27.08.2010-08.05.2015 & FO \\
\hline & & $\begin{array}{l}\text { Internetauftritte und Facebook-Profile von Bürgerinitiativen } \\
\text { im Kontext des Stromnetzausbaus }\end{array}$ & $\begin{array}{l}\text { Dezember 2014-Januar } \\
2015\end{array}$ & BI \\
\hline & & Interviews & März-April 2015 & \\
\hline & & BMWi (Bundesministerium für Wirtschaft und Energie) & & IP01 \\
\hline & & $\begin{array}{l}\text { BMUB (Bundesministerium für Umwelt, Naturschutz, Bau } \\
\text { und Reaktorsicherheit) }\end{array}$ & & IP02 \\
\hline & & BNetzA (Bundesnetzagentur) & & IP03 \\
\hline & & Amprion $\mathrm{GmbH}$ & & $\mathrm{IP} 04+05$ \\
\hline & & TenneT TSO GmbH & & IP06 \\
\hline & & DUH (Deutsche Umwelthilfe e. V.) & & IP11 \\
\hline & & Mitarbeiter Bürgerbeteiligungsprozess Demoenergie & & IP12 \\
\hline & & Mediziner, Experte für elektrische und magnetische Felder & & IP13 \\
\hline & & NABU (Naturschutzbund Deutschland) & & IP15 \\
\hline & & Systemanalytiker Netzausbau & & IP16 \\
\hline \multirow{16}{*}{$\begin{array}{l}\text { Nord- } \\
\text { deutschland }\end{array}$} & \multirow[t]{4}{*}{ Landesebene } & Interviews & März-April 2015 & \\
\hline & & $\begin{array}{l}\text { NMU (Niedersächsisches Ministerium für Umwelt, Energie } \\
\text { und Klimaschutz) }\end{array}$ & & IP08 \\
\hline & & $\begin{array}{l}\text { NLWKN (Niedersächsischer Landesbetrieb für Wasserwirt- } \\
\text { schaft, Küsten- und Naturschutz) }\end{array}$ & & IP09 \\
\hline & & $\begin{array}{l}\text { Stimme aus dem ministeriellen Bereich Nordrhein-Westfa- } \\
\text { lens }\end{array}$ & & IP10 \\
\hline & \multirow{6}{*}{$\begin{array}{l}\text { „Keine } 380 \mathrm{kV} \text { - } \\
\text { Freileitung am } \\
\text { Teuto“ }\end{array}$} & $\begin{array}{l}\text { Webinhalt Bürgerinitiative „Keine } 380 \text { kV-Freileitung am } \\
\text { Teuto“ }\end{array}$ & März-April 2015 & Mat-lok09 \\
\hline & & $\begin{array}{l}\text { Flyer, Infomaterial, Erklärungen Bürgerinitiative „Keine } \\
380 \text { kV-Freileitung am Teuto“ }\end{array}$ & März-April 2015 & Mat-lok10 \\
\hline & & $\begin{array}{l}\text { Trassendialog Lüstringen - Hesseln. Gemeinsame Erklä- } \\
\text { rung unter Beteiligung der Bürgerinitiative „Keine } 380 \mathrm{kV} \text { - } \\
\text { Freileitung am Teuto“ }\end{array}$ & März-April 2015 & Mat-lok11 \\
\hline & & $\begin{array}{l}\text { Neue Westfälische (NW) } \\
\text { http://www.nw.de }\end{array}$ & 04.09.2014-12.04.2015 & N-lok07 \\
\hline & & $\begin{array}{l}\text { Westdeutsche Allgemeine Zeitung (WAZ) } \\
\text { http://www.derwesten.de }\end{array}$ & $31.10 .2012-10.04 .2015$ & N-lok08 \\
\hline & & $\begin{array}{l}\text { Interviews mit einem Kommunalvertreter aus Hilter am Teu- } \\
\text { toburger Wald sowie der Bürgerinitiative }\end{array}$ & März 2015 & $\begin{array}{l}\text { IP-lok07 } \\
\text { IP-lok08 }\end{array}$ \\
\hline & \multirow[t]{6}{*}{$\begin{array}{l}\text { „Delligsen in der } \\
\text { Hilsmulde“ }\end{array}$} & $\begin{array}{l}\text { Internet-Kommunalinformationen Delligsen, Informationen } \\
\text { rund um die „Hamelner Erklärung“ }\end{array}$ & März-April 2015 & Mat-lok06 \\
\hline & & Webinhalt Bürgerinitiative „Delligsen in der Hilsmulde“ & März-April 2015 & Mat-lok07 \\
\hline & & $\begin{array}{l}\text { Flyer, Infomaterial Bürgerinitiative „Delligsen in der Hils- } \\
\text { mulde“ }\end{array}$ & März-April 2015 & Mat-lok08 \\
\hline & & $\begin{array}{l}\text { Einbecker Morgenpost } \\
\text { https://www.einbecker-morgenpost.de/einblicke.html }\end{array}$ & 22.07.2010-17.07.2014 & N-lok05 \\
\hline & & $\begin{array}{l}\text { Hessische Niedersächsische Allgemeine (hna) http://www. } \\
\text { hna.de }\end{array}$ & $25.10 .2010-09.03 .2015$ & N-lok06 \\
\hline & & $\begin{array}{l}\text { Interviews mit einem Kommunalvertreter aus Delligsen so- } \\
\text { wie der Bürgerinitiative }\end{array}$ & März 2015 & $\begin{array}{l}\text { IP-lok05 } \\
\text { IP-lok06 }\end{array}$ \\
\hline
\end{tabular}


Tab. 1 Übersicht über die Analysebestandteile (Fortsetzung)

\begin{tabular}{|c|c|c|c|c|}
\hline \multirow{2}{*}{$\frac{\text { Ebene }}{\text { Bayern }}$} & & Medium/Interviewpartner & Zeitpunkt/Zeitraum & Kürzel \\
\hline & \multirow[t]{3}{*}{ Landesebene } & Interviews & März-April 2015 & \\
\hline & & $\begin{array}{l}\text { StMWi (Bayerisches Staatsministerium für Wirtschaft und } \\
\text { Medien, Energie und Technologie) }\end{array}$ & & IP07 \\
\hline & & Bund Naturschutz Bayern e. V. & & IP14 \\
\hline & \multirow[t]{6}{*}{$\begin{array}{l}\text { „Pegnitz unter } \\
\text { Strom“ }\end{array}$} & $\begin{array}{l}\text { Internet-Kommunalinformationen Pegnitz sowie Reden und } \\
\text { Schreiben des Bürgermeisters gegen die Gleichstrompassage, } \\
\text { Pegnitzer Resolution zur Energiewende, Informationen zum } \\
\text { Verein „Kommunen gegen die Gleichstrompassage“ }\end{array}$ & März-April 2015 & Mat-lok03 \\
\hline & & Webinhalt Bürgerinitiative „Pegnitz unter Strom“ & März-April 2015 & Mat-lok04 \\
\hline & & Flyer, Infomaterial Bürgerinitiative „Pegnitz unter Strom“ & März-April 2015 & Mat-lok05 \\
\hline & & $\begin{array}{l}\text { Frankenpost - Tageszeitung für Oberbayern } \\
\text { http://www.frankenpost.de }\end{array}$ & 27.12.2012-03.02.2015 & N-lok03 \\
\hline & & $\begin{array}{l}\text { Nürnberger Land (Hersbrucker Zeitung, Pegnitz-Zeitung und } \\
\text { Der Bote) } \\
\text { http://www.n-land.de }\end{array}$ & 15.10.2012-22.02.2015 & N-lok04 \\
\hline & & $\begin{array}{l}\text { Interviews mit einem Kommunalvertreter aus Pegnitz sowie } \\
\text { der Bürgerinitiative }\end{array}$ & März 2015 & $\begin{array}{l}\text { IP-lok03 } \\
\text { IP-lok04 }\end{array}$ \\
\hline & \multirow[t]{5}{*}{$\begin{array}{l}\text { „Hormersdorf- } \\
\text { Schnaittach“ }\end{array}$} & $\begin{array}{l}\text { Internet-Kommunalinformationen Markt Schnaittach } \\
\text { („Gleichstrompassage“ auf Startseite positioniert) }\end{array}$ & März-April 2015 & Mat-lok01 \\
\hline & & Webinhalt Bürgerinitiative „Hormersdorf-Schnaittach“ & März-April 2015 & Mat-lok02 \\
\hline & & $\begin{array}{l}\text { Mein Mitteilungsblatt Schnaittach } \\
\text { http://www.mein-mitteilungsblatt.de/schnaittach/themen/ } \\
\text { hormersdorf.html }\end{array}$ & 09.01.2013-18.01.2015 & N-lok01 \\
\hline & & $\begin{array}{l}\text { Online-Portal Nürnberger Zeitung und Nürnberger Nachrich- } \\
\text { ten } \\
\text { http://www.nordbayern.de }\end{array}$ & $30.05 .2012-09.03 .2015$ & N-lok02 \\
\hline & & $\begin{array}{l}\text { Interviews mit einem Kommunalvertreter aus Schnaittach } \\
\text { sowie der Bürgerinitiative }\end{array}$ & März 2015 & $\begin{array}{l}\text { IP-lok01 } \\
\text { IP-lok02 }\end{array}$ \\
\hline
\end{tabular}

GT Google-Treffer, SZ Süddeutsche Zeitung, FO Focus, BI Bürgerinitiative, IP Interviewpartner,

Mat-lok Materialien der lokalen Ebene, das heißt, Internetseiteninhalte sowie Flyer, Postkarten etc.,

N-lok Online frei verfügbare (Zeitungs-)Artikel auf regionaler bzw. lokaler Ebene. Die unterschiedlichen Zeiträume bei den Artikeln ergeben sich aus abweichenden Zeiträumen vorhandener Artikel innerhalb der Onlineportale der Medien,

IP-lok Interviewpartner auf der Ebene der Fallstudien

als schnelle und unkomplizierte Möglichkeit der Recherche zu und gerade hoch gerankte Google-Treffer werden häufig zum ersten Zugang zu Informationen (Pan/Hembrooke/ Joachims et al. 2007; vgl. Schmidt 2011). Entsprechend wurden zur Berücksichtigung medialer Darstellungen die ersten 50 Treffer der Suchmaschine Google zum Schlagwort „Stromnetzausbau“ zusammengestellt und analysiert (GT01 bis GT50; Google ist in Deutschland unangefochtener Marktführer der Suchmaschinen). Um den medialen Diskurs zum Stromnetzausbau mit nationaler Schwerpunktsetzung abzubilden, wurden alle online frei zugänglichen und damit vom Grundsatz her breit rezipierbaren Artikel des Marktführers bei überregionalen Zeitungen, der Tageszeitung Süddeutsche Zeitung (SZ), und des Wochenmagazins mit der größten Internetreichweite, dem Focus (FO), zum Schlagwort „Netzausbau“ (mit Bezug zum Stromnetzausbau, unberücksichtigt blieben damit unter anderem Beiträge um den Ausbau der Mobilfunknetze) in Deutschland zusammengestellt, die einen Zeitraum zwischen Mai/August 2010 und April/Mai 2015 abdecken (systematisiert als SZ und FO jeweils mit Angabe des Erscheinungsdatums). Mit Google-Treffern sowie $S Z$ und Focus gehen wir über bisherige mediendiskursanalytische Ansätze hinaus, die sich zumeist auf ein Medium beschränkten (vgl. z. B. Brailich/ Germes/Schirmel et al. 2008; Schirmel 2011; Kühne 2012; Glasze/Weber 2014).

Aktuelle Einschätzungen und Bewertungen wurden durch 16 qualitative episodische Interviews ${ }^{2}$ mit Vertretern von Institutionen und Initiativen erfasst. Gleichzeitig wurden darüber Argumentationsmuster im Detail analysiert und auch solche Muster beleuchtet, die medial weniger dominieren. Die Narrationen der Interviewpartner können diskurstheoretisch als ,artikulatorische Akte“ (Nonhoff 2006: 185) aufgefasst werden, die diese als Teil von Institutionen

\footnotetext{
2 Entsprechend den Prämissen der Diskurstheorie wurden möglichst offene Interviews mit langen Erzählpassagen geführt. Um durch die Weite des Feldes keine Verlorenheit zu produzieren, wurden Fragen als Anstöße gestellt, wodurch Erzählepisoden zu einzelnen Aspekten entstanden (vgl. auch Weber 2013: 71 ff.).
} 
und Initiativen - damit in deren Namen - als Sprecher im Diskurs vornehmen. Wird berücksichtigt, dass immer mehrere Diskursstränge parallel existieren, leitet sich daraus auch ab, dass Befragte in der Regel nicht nur einen Diskurs wiedergeben, sondern Stränge unterschiedlicher Diskurse einfließen (Mattissek 2005: 120; Weber 2013: 60 f.). Neben Mitarbeitern aus Bundes- und Landesministerien, der Bundesnetzagentur und der Übertragungsnetzbetreiber wurden Experten aus dem Bereich der Dialoggestaltung, von Umwelt- und Naturschutzverbänden, der Medizin und der Energiesystementwicklung befragt (IP01 bis IP16).

Zur Erfassung bürgerschaftlichen Engagements wurden mittels Google Bürgerinitiativen, die sich im Zuge des Stromnetzausbaus konstituiert haben und über eine eigene Website oder ein Facebook-Profil verfügen, recherchiert und deren Profile nach weiteren genannten Initiativen durchsucht. Auf diese Weise wurden 90 Bürgerinitiativen ermittelt und deren angebotene Informationen auf zentrale Positionierungen hin untersucht.

Um zu überprüfen, ob sich regionale und lokale Bezugnahmen von eher nationalen oder länderbezogenen unterscheiden, wurden diskursive Aushandlungsprozesse rund um vier Bürgerinitiativen analysiert. Ausgehend vom starken Ausbau erneuerbarer Energien im Norden, deren Strom in den industriereichen Süden Deutschlands transportiert werden soll (vgl. Übertragungsnetzbetreiber 2014a; Übertragungsnetzbetreiber 2014b), wurden in Relation zur Analyse der 90 Bürgerinitiativen mit Webprofil zwei Fallstudien aus Niedersachsen und Nordrhein-Westfalen sowie zwei aus Bayern ausgewählt. Zu den Fallstudien ,Keine 380 kVFreileitung im Teuto“ (Zusammenschluss von Initiativen mehrerer Orte ,,unter einem Dach“), „Delligsen in der Hilsmulde“ (seit 2008 bestehend und damit mit langer „Protesterfahrung" (Marg/Geiges/Butzlaff et al. 2013)) (beide Norddeutschland), „Pegnitz unter Strom“ (starke Verknüpfung Bürgerinitiative und Stadt Pegnitz sowie Stadt Pegnitz im Verein „Kommunen gegen die Gleichstrompassage SüdOst“ engagiert) und „Bürgerinitiative Hormersdorf-Schnaittach" (Markt Schnaittach nicht in vorgenanntem Verein engagiert) (beide Bayern) wurden Artikel aus zwei Zeitungen pro Fallkontext - jeweils zum einen eher regional und zum anderen lokal - untersucht (Zeiträume Juli 2010 bis März 2015, N-lok01 bis N-lok08), jeweils ein hoher kommunaler Vertreter und Mitglieder der Bürgerinitiativen befragt (IP-lok01 bis IP-lok08) sowie die Internetinformationen der Kommunen und Bürgerinitiativen und ergänzende Materialien ausgewertet (Mat-lok01 bis Mat-lok11). Innerhalb der nachfolgenden Analyse werden synthetisierend zentrale Argumentationsmuster, insbesondere der Kritik, und regionale Unterschiede herausgearbeitet.

\section{Ergebnisse: Konstituierung von „Energiewende“" und zentrale Knotenpunkte der Ablehnung von Planungen}

\subsection{Diskursive Grundlagen des Stromnetzausbaus, die „Energiewende ${ }^{66}$ und die ,Bedarfsfrage ${ }^{66}$}

Die Diskursstränge um Energiewende und Stromnetzausbau leiten sich - im Rahmen des Beitrags aus Platzgründen entkomplexisierend - aus gesamtgesellschaftlichen Diskursen ab: Klimawandel, Energiepreisentwicklungen, Energieautarkie und Kernkraft sind eng mit der Energiewende und damit auch mit dem Netzausbau verbunden (vgl. Abb. 2). Auch wenn von einigen Stimmen in der medialen Berichterstattung - Google, Süddeutsche Zeitung und Focus - der Klimawandel als ,Erfindung“ von Wissenschaft und Politik gerahmt wird, ist dieser hegemonial als „Fakt" verankert, auf den zu reagieren sei, gerade mit dem Ausbau erneuerbarer Energien und damit dem Ausbau bestehender Stromnetze (z. B. SZ-12.06.2011, SZ-28.08.2012). Wird davon ausgegangen, dass im Zuge einer Verknappung fossiler Energieträger erneuerbare Energien konkurrenzfähiger werden und im Zeitalter wachsender Vernetzung nationale Energieautarkie im Gegensatz zu europäischen Stromnetzen nur schwerlich umsetzbar erscheint, so wird auch hierüber der Stromnetzausbau als erforderlich diskursiv (re)produziert. Nach Fukushima gehört es - Stichwort „Risiken der Kernenergienutzung“ - kaum noch zum Bereich des „Sagbaren“ (in Anschluss an Michel Foucault, vgl. Weber 2013: 61 ff.), die weitere zivile Nutzung der Kernkraft zu favorisieren („Sicherheitsdiskurs“ nach Leibenath/Otto 2012: 129). Die Folgen eines Reaktorunfalls seien nicht vertretbar, ein Ausstieg aus der Kernkraft entsprechend zwingend erforderlich, die Herausforderungen einer Endlagerung hochradioaktiver Abfälle nicht gelöst (z. B. GT08, FO-17.02.2014).

Die skizzierten Argumentationsstränge führen - tendenziell übergreifend geteilt - zur Bewertung, die Energiewende sei notwendig - die Energiewende als zentraler diskursiver Knotenpunkt. Dass nun damit allerdings auch ein umfänglicher Bedarf des Ausbaus bestehender Stromnetze bestehe, wird nicht durchgehend geteilt (Konfliktfeld Bedarfsfrage, vgl. auch Abb. 2). Die Infragestellung der Notwendigkeit erfolgt - tendenziell kognitiv argumentierend besonders mit dem Hinweis darauf, dass die Berechnungen in den Netzentwicklungsplänen nicht nachvollziehbar seien bzw. befürchtet würde, über die Trassen würde Kohlestrom aus Ostdeutschland und Atomstrom aus dem Ausland transportiert (vgl. auch Jarass/Obermair 2012; Neukirch 2014; Neukirch 2015) - dazu beispielhaft: Der Bund für Umwelt und Naturschutz Deutschland (BUND) lehne die Trassen $\mathrm{ab}$, ,solange deren Notwendigkeit [...] nicht nachgewiesen und nachvollziehbar begründet wurde" (GT30). Der Netzausbau diene dem ,Transport von Braunkohlestrom zeit- 
Abb. 2 Diskursive Aushandlungsprozesse - Synthetisierende, entkomplexisierende Darstellung zentraler Argumentationszusammenhänge, Aushandlungsprozesse insbesondere um die Bedarfsfrage des Netzausbaus (rot hervorgehoben) sowie im Hinblick auf die angeführten Konfliktfelder (dunkler schattiert). (Eigene Darstellung auf der Basis der Diskursanalysen der Google-Treffer, SZund Focus-Artikel, der geführten Interviews sowie der Fallstudien (vgl. Tab. 1). Umsetzung Tobias Sontheim (2016))

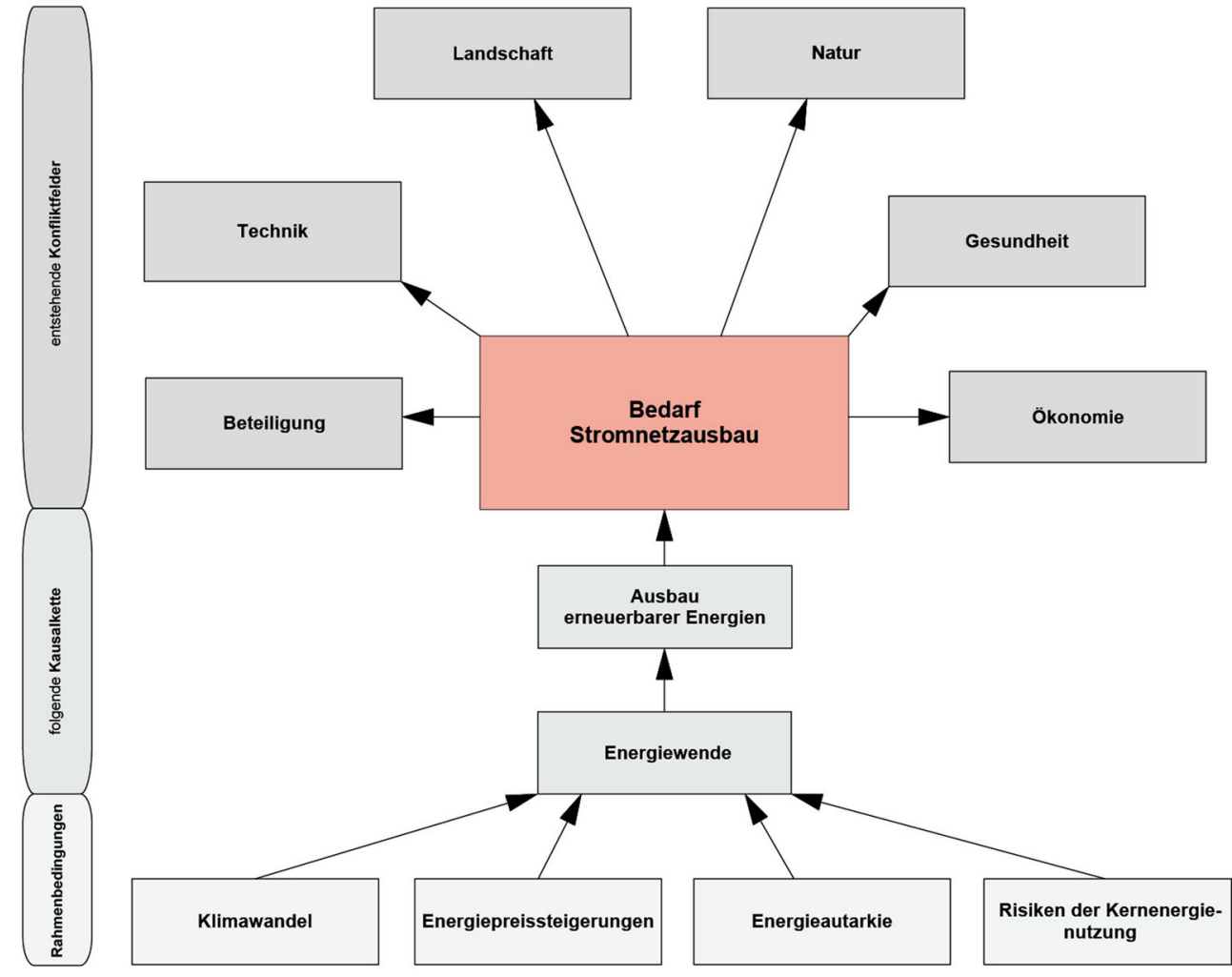

gleich zu Starkwindeinspeisungen in Ostdeutschland" und eröffne zusätzlich die Möglichkeit, „dass Atomstrom eingespeist wird" (IP16 Systemanalytiker). Entsprechende Argumentationsmuster werden tendenziell - regional gesehen - im Freistaat Bayern (re)produziert. Dort wird zusätzlich regelmäßig über das Streben nach einer dezentralen, regionalen Energieversorgung argumentiert. Der Ausbau erneuerbarer Energien sei für die „bayerische Energiewende“ vorangetrieben worden, dem nun die geplanten neuen Trassen zuwiderliefen, weswegen sich Widerstand rege (unter anderem IP14 Bund Naturschutz Bayern, IP16 Systemanalytiker). Die Energiewende wird dort mit dezentraler, regionaler Energieversorgung verwoben, womit der geplante Stromnetzausbau hier regional zum Außen des „Energiewende"-Diskurses wird. Damit wird gleichzeitig Energiewende zu einem „flottierenden Signifikanten“ (Laclau 2007: 131), also einem Knotenpunkt, der an unterschiedliche Diskursstränge anschlussfähig ist: Energiewende - im nationalen Diskurs - in Verbindung mit dem Stromnetzausbau gegenüber dezentraler, bayerischer Energiewende. Auch in den bayerischen Fallstudien „Pegnitz unter Strom“ und „Hormersdorf-Schnaittach“ sind Narrationen einer regionalen Energiewende und einer dezidierten Ablehnung geplanter Stromtrassen, hier speziell der Gleichstrompassage Süd-Ost, hegemonial verankert. Kommunalvertreter (IPlok03) und ein Mitglied der Bürgerinitiative in Pegnitz führen unter anderem an, „dezentrale Versorgungselemente“
(IP03) seien zu stärken und ,wir brauchen diese Stromtrasse auf keinen Fall“" (IP-lok04). In Schnaittach heißt es: ,wir sehen diese Trassen als völlig kontraproduktiv für diese Energiewende" an (IP-lok02 Bürgerinitiative). Von 38 in Bayern mit Internet- oder Facebook-Auftritt ermittelten Bürgerinitiativen (90 auf Deutschlandebene, vgl Abschn. 2.3). sprachen sich im Januar 2015 insgesamt 37 grundsätzlich gegen neue Stromtrassen aus. Auch die ermittelten hessischen (8), baden-württembergischen (5) und thüringischen (4) Bürgerinitiativen lehnen die Trassenplanungen ab (vgl Abb. 3). - tendenziell also ein Schwerpunkt Süddeutschland/Südostdeutschland.

Aushandlungsprozesse um das „Wie“ des Netzausbaus werden durch die Grundsätzlichkeit der Ablehnung erschwert bzw. fast unmöglich. Auffällig ist auch, wie bereits in der Einleitung angedeutet, der starke Vernetzungsgrad von Bürgerinitiativen gegen den Stromnetzausbau, was auch die Durchführung gemeinschaftlicher Aktionen einschließt. So fanden in Bayern ab Februar 2014 mehrere „Trassenkonferenzen“, Treffen und Aktionstage statt. Das Handeln der bayerischen Staatsregierung zeigt sich stark mit dem Agieren der Initiativen rückgekoppelt: Forderte sie noch im Oktober 2013 einen stärkeren Netzausbau, wurden zwischenzeitlich ab Oktober 2014 neue Stromtrassen abgelehnt, bis im Juli 2015 Erdverkabelungen statt Freileitungen vereinbart wurden (vgl. Abb. 4). Der massive Protest bayerischer Bürgerinitiativen blieb nicht folgen- 


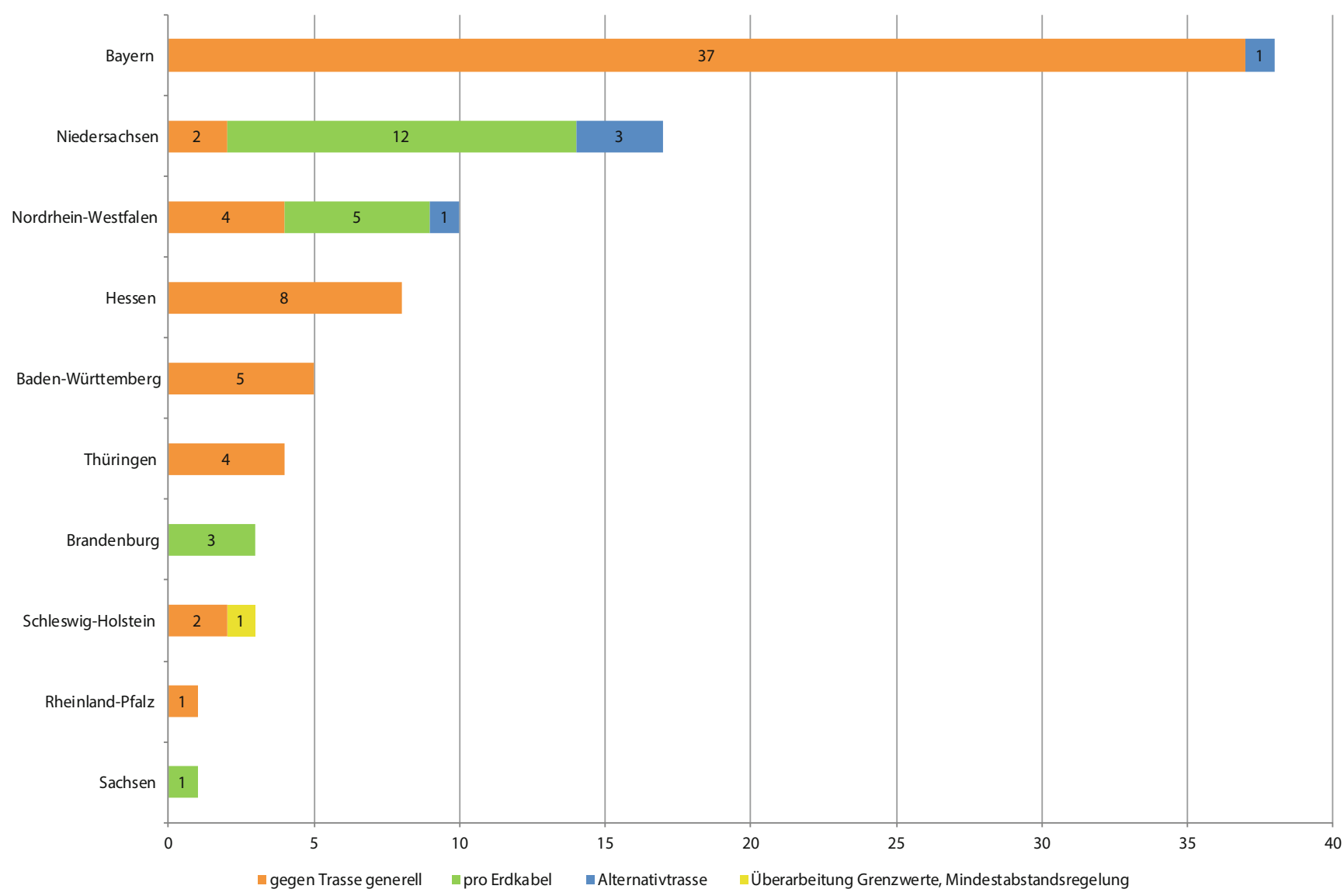

Abb. 3 Ziele der Bürgerinitiativen nach Ländern. (Weber/Jenal/Kühne 2016: 54)

los für politische Entscheidungen und das Agieren der bayerischen Staatsregierung beförderte die Intensität des Widerstandes der Bürgerinitiativen.

Der grundsätzlich ablehnenden Haltung mit regionalem Schwerpunkt Süddeutschland/Südostdeutschland fallstudienbezogen mit Schwerpunkt Bayern untersucht können Diskursstränge mit eher nationalem und norddeutschem Schwerpunkt gegenübergestellt werden, in denen in erster Linie kognitiv über betriebswirtschaftlich-technische Notwendigkeiten argumentiert wird. Die bestehenden Stromnetze reichten nicht aus, um ohne vielfältige Eingriffe Versorgungssicherheit zur gewährleisten. „Bund, Länder[-] und Netzbetreiber[-]“ konstatierten unter anderem, dass der Netzausbau „benötigt“ werde (GT15) beziehungsweise wird der Netzausbau als „Voraussetzung“ beschrieben, um den weiteren Ausbau erneuerbarer Energien gewährleisten zu können (GT16, vergleichbar GT35). Wer die Energiewende wolle, müsse „den Strom dort produzieren, wo es am günstigsten“ sei, dies sei „beim Wind in Norddeutschland der Fall. Es muss dann aber auch der Transport nach Süddeutschland" ermöglicht werden (FO07.10.2014). Innerhalb der in den Google-Treffern, den SZ- und den Focus-Artikeln vertretenen Sprecherpositionen ist die Befürwortung des Netzausbaus zur Gewährleistung der Energiewende recht stark verankert. $66 \%$ (Google), $67 \%$ (SZ) und $76 \%$ (Focus) der Sprecherpositionen votieren für die Umsetzung vorliegender Trassenplanungen, ${ }^{3}$ wobei hier gerade Bundes- und Landespolitiker, Netzbetreiber und die Bundesnetzagentur, aber deutlich weniger Bürgerinitiativen $\mathrm{zu}$ Wort kommen. Letztere finden in den regionalen und vor allem den lokalen Zeitungen der Fallstudien mittlere bis starke Berücksichtigung (vgl. ausführlich Weber/Kühne/Jenal et al. 2015: 82 ff.). Gegen die Trassenplanungen votieren jeweils weniger als ein Viertel der Sprecher bei Google, SZ und Focus. Ein Eintreten für Erdkabel ist hier maximal als ein Subdiskurs zu werten. 25 der 33 ermittelten Bürgerinitiativen aus Niedersachsen, Nordrhein-Westfalen, Brandenburg und SchleswigHolstein - vereinfachend „norddeutsche“ - sprechen sich wiederum für Erdverkabelungen, alternative Trassenführungen oder Überarbeitungen der bestehenden Grenzwerte bzw. für Mindestabstandsregelungen aus, während nur acht gänzlich die Trassenplanungen ablehnen (vgl. Abb. 3).

\footnotetext{
3 In den Google-Treffern wurden 77, in den SZ-Artikeln 135 und in den Focus-Artikeln 108 Sprecherpositionen erfasst, die den Feldern „pro Trasse“, ,pro Erdkabel“, ,erneute Prüfung“ und „gegen Trasse“ zugeordnet wurden.
} 


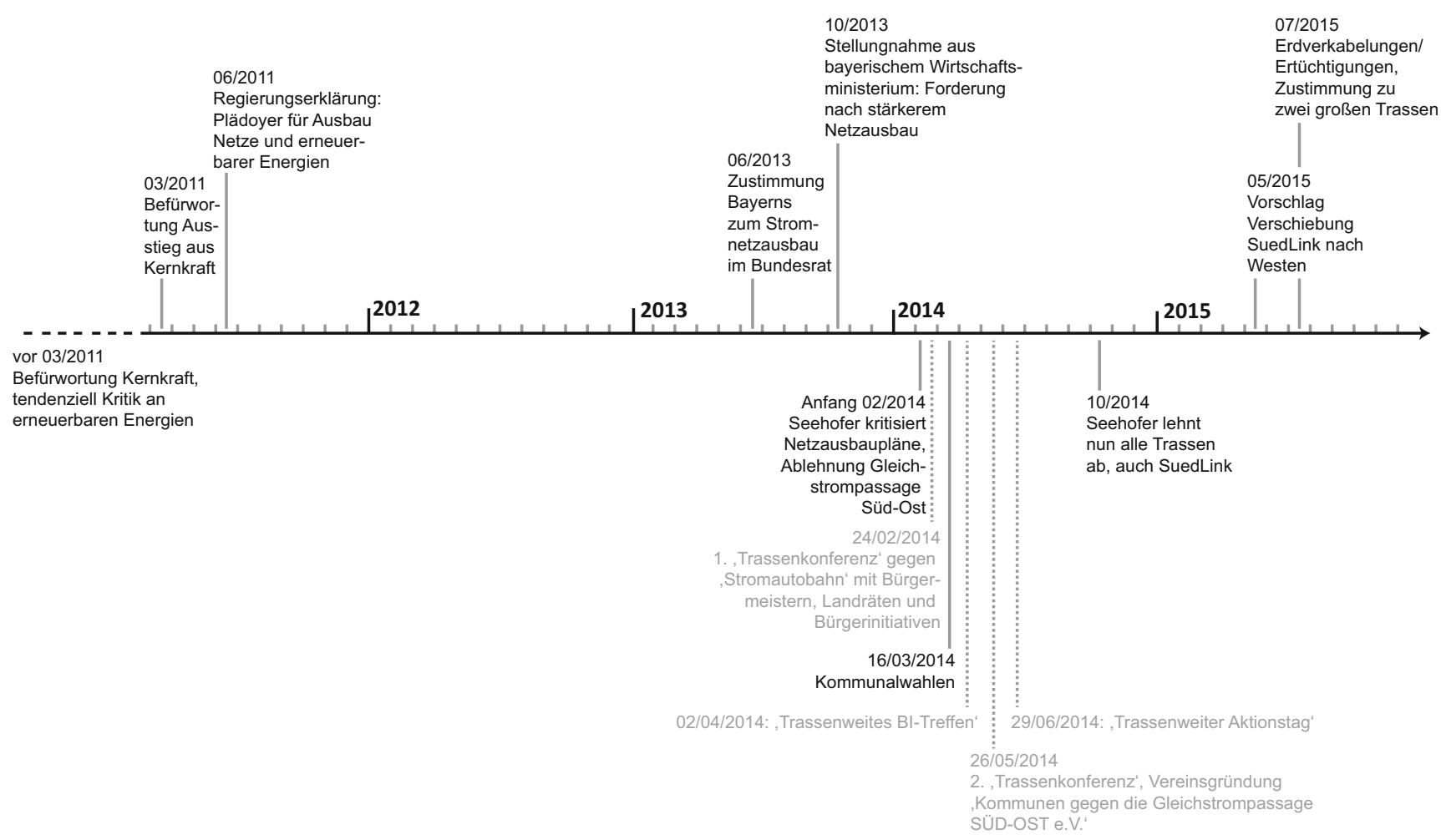

Abb. 4 Markante Positionswechsel der bayerischen Staatsregierung (Befürwortung versus Ablehnung Kernkraft sowie Zustimmung versus Ablehnung Stromnetzausbauplanungen). (Weber/Kühne/Jenal et al. 2016: 84, leicht verändert)

In den geführten Interviews wird die tendenzielle Befürwortung des Stromnetzausbaus im nationalen Mediendiskurs und bei den norddeutschen Bürgerinitiativen in Verbindung mit der Energiewende unterstrichen. Ohne den Stromnetzausbau sei die Energiewende mit dem Ausbau der erneuerbaren Energien ,gar nicht zu schaffen“ (IP04 Amprion) bzw. die Energiewende erfordere, Netze „umzubauen und auszubauen“ (IP06 TenneT). Diskurstheoretisch interpretiert entsteht eine Äquivalenzkette aus Energiewende, erneuerbaren Energien und Stromnetzausbau. Im Gegensatz zu den bayerischen ist die dargestellte Verknüpfung in den norddeutschen Fallstudien Teil des lokalen Diskurses. Vertreter der Bürgerinitiative ,Keine 380 kV-Freileitung am Teuto“ (Grenzbereich Niedersachsen/Nordrhein-Westfalen) akzeptieren die Koppelung aus Energiewende und NordSüd-Stromtransport: Die „Notwendigkeit der Energiewende, das ist nicht in Frage gestellt", ,niemand würde sagen, wir brauchen keine Stromnetze“, begründet darüber, dass „Windstrom aus dem Norden nach Süden muss“ (IPlok08). Dieses Muster findet sich auch bei der Fallstudie „Delligsen in der Hilsmulde“, beispielsweise (re)produziert durch einen Kommunalvertreter (IP-lok05): „wir brauchen die Energie ja und wir müssen die Energie auch transportieren und deswegen brauchen wir auch die Stromautobahnen, sag ich mal. [...]. Wenn das nicht gelingt, dann kriegen wir auch diese Wende nicht hin." Der Stromnetzausbau als Konsequenz der Energiewende ist hegemonial verankert, was jedoch nicht zur Folge hat, dass auch bisherige Planungen ,einfach“ akzeptiert würden. Als zentrale Konfliktfelder konnten Beteiligung, Technik und Gesundheit, Ökonomie sowie Natur und Landschaft herausgearbeitet werden (vgl. auch Abb. 2), die im Folgenden beleuchtet werden.

\subsection{Ablehnung von Planungen durch spezifische Bezugnahmen}

\subsubsection{Unzureichende Beteiligung}

Fragen der Bürgerbeteiligung erfahren eine sehr unterschiedliche Einschätzung. Auf der einen Seite wird auf Informationsveranstaltungen und Dialogprozesse rekurriert, die dazu dienten, einen ,möglichst breiten gesellschaftlichen Konsens“ zu erzielen (GT10). Die Bundesnetzagentur veranstaltet seit Herbst 2012 Informationsveranstaltungen (,Infotage“) zur Information der Öffentlichkeit: „,Wir haben von Anfang an geplant, bewusst eine breite Öffentlichkeit mitzunehmen, breiter als es gesetzlich vorgegeben ist" (IP03). Die Übertragungsnetzbetreiber führen Informations- und Dialogveranstaltungen sowie „Runde Tische“ an, bei denen diese „Rede und Antwort stehen“ (IP04 Amprion) und Bürger einbeziehen würden (IP06 TenneT). Grundsätzlich besteht Konsens, Bürgerbeteiligung zur Umsetzung von Planungszielen sei unumgänglich, wie eine Narration des Ministerpräsidenten von Schleswig-Holstein, Torsten 
Albig, beispielhaft zeigt: „Der Netzausbau gelingt nur, wenn wir die Menschen in der Region dabei mitnehmen“" (FO-30.01.2013). Der Ansatz ,frühzeitiger“ Information und das Einräumen von Mitgestaltungsmöglichkeiten wird auf fast identische Weise von unterschiedlichen Sprechern - Ministerien, Deutsche Umwelthilfe (DUH), Übertragungsnetzbetreiber - (re)produziert und auf diese Weise zu einem festen Bestandteil aktueller Diskussionskultur - als hegemonial diskursiv verankert zu deuten.

Auf der anderen Seite werfen Landesregierungen und Bürgerinitiativen den Verantwortlichen allerdings auch mangelnde Transparenz und Beteiligungsmöglichkeiten vor (SZ-04.02.2014 und SZ-23.02.2013). Mitarbeiter der Bundesnetzagentur merken hierzu an, die Übertragungsnetzbetreiber schienen von starker Beteiligung und Protest überrascht gewesen zu sein. Diese seien ,,vielleicht erst einmal nach altbewährtem Muster an die Sache herangegangen“, was sich aber gewandelt habe: „Die Transparenz oder Informationsbereitschaft der Übertragungsnetzbetreiber hat sich positiv entwickelt" (IP03). In diese Richtung argumentiert auch ein Mitarbeiter von TenneT: „Wir haben unsere Arbeit so umgestellt, dass wir sehr früh informieren“ (IP06). Als Problematik wird mehrfach angeführt, in Dialogveranstaltungen hätten Bürger zwar die Möglichkeit, ihre Wünsche zu äußern, aber erst im offiziellen Planfeststellungsverfahren lägen endgültig konkrete Trassenverläufe vor und erst in diesem könne ,der Bürger dann seine mögliche konkrete Betroffenheit endgültig einschätzen“ (IP03 BNetzA, ähnlich IP02 BMUB, IP04 Amprion; zu den Problematiken von Bürgerbeteiligung beim Stromnetzausbau vgl. auch ausführlich Stegert/Klagge 2015: $181 \mathrm{ff}$.).

Die Untersuchungen der Bürgerinitiativen zeigen in verschiedenen, vergleichbaren narrativen Mustern Bezüge auf fehlendes Vertrauen gegenüber den Übertragungsnetzbetreibern, Politik und Planung. Diese hätten zu Planungsbeginn unzureichend informiert und fehlendes Vertrauen könne kaum wiederhergestellt werden, wie nachfolgende Passagen der Fallstudien „Keine 380 kV-Freileitung am Teuto“ und „Pegnitz unter Strom“ verdeutlichen: „Die Fehler wurden am Anfang gemacht, da wurde nicht ausreichend, auch nicht auf Augenhöhe informiert, sondern von oben herab." Wenn Vertrauen nicht mehr gegeben sei, ,ist es unheimlich schwer, da wieder ein Vertrauen reinzubringen“ (IP-lok07 Kommunalvertreter Hilter, Kontext „Keine $380 \mathrm{kV}$-Freileitung am Teuto“). Unzufriedenheit drückt sich in emotionalen Bezugnahmen aus: „Amprion hat eine Veranstaltung gemacht, die noch zwei Mal wiederholt wurde. Skandalös war's. Skandalös, weil von hinten bis vorne mit Unwahrheiten informiert wurde und wenn man dann konkrete Fragen gestellt hat, dann ist man denen sehr, sehr ungeschickt ausgewichen.“ Amprion mache ,es sich sehr, sehr einfach [...]. Es gibt ein Gesetz und das ist die Grund- lage für ihre Tätigkeit. Und das Gesetz hat sich nicht geändert und so lange wie es sich nicht ändert, haben sie den Auftrag“ (IP-lok04 Bürgerinitiative „Pegnitz unter Strom“). Der Bezug auf gesetzliche Rahmenbedingungen wird eher als Ausrede denn als stichhaltiges Argument gewertet. Bürgerbeteiligung wird als unzureichend (re)produziert, womit Planungsprozesse delegitimiert werden. Diskurstheoretisch gefasst ist Beteiligung als wünschens- und erstrebenswert hegemonial verankert, allerdings besteht Dissens in Bezug auf deren Erfüllung.

\subsubsection{Begrenztes Vertrauen in neue Technik und gesundheitliche Befürchtungen}

Als konkrete Argumente, die gegen bestehende Planungen vorgebracht werden bzw. die als solche wahrgenommen werden und damit in Diskurssträngen um den Stromnetzausbau mitschwingen, wird regelmäßig auf die technische Umsetzung rekurriert, was vielfach in engem Zusammenhang mit gesundheitsbezogenen Befürchtungen steht. Unter anderem der SuedLink und die Gleichstrompassage Süd-Ost werden in Hochspannungsgleichstromübertragungstechnik (HGÜ) geplant - einer bisher in Deutschland noch nicht eingesetzten Technik, die mit ,viel größeren Beschwerden, [...] viel größeren Ängsten“ durch die neue Technik verbunden würde, so beispielsweise ein AmprionMitarbeiter (IP04) (allgemein zu Fragen von Technikakzeptanz vgl. Renn 2005). Im Mittelpunkt stehen ,so genannte[-] Korona-Effekte [...], die bei Regen oder Schnee Lärmbeziehungsweise Geräuschpegel verursachen können“ und „wo sich ionisierte Raumladungswolken über wenige Kilometer hinweg verteilen und noch messbar sind. [...]. Nach derzeitigem Stand der Wissenschaft sind durch Ionenwolken keine gesundheitlichen Auswirkungen auf den menschlichen Organismus zu erwarten“ (IP03 BNetzA). Ein befragter Mediziner (IP13) führt dagegen an, ,Staub und andere Partikel“ könnten sich ,kumulierend ansammeln und so eine Art zusätzliche Belastung für die Bevölkerung ergeben“. Durch mehrere Interviewpartner wird ein bisher bestehendes Forschungsdefizit zur HGÜ-Technik angeführt (IP03 BNetzA, IP10 Ministeriumsebene Nordrhein-Westfalen). So wird auch durch den Kommunalvertreter aus Pegnitz (IP-lok04) der Einsatz dieser Technik mit dem Verweis auf gesundheitliche Befürchtungen strikt abgelehnt: „Wir sprechen über eine neuartige Technik, da bin ich jetzt gleich mal schon in der kritischen Betrachtung dieser geplanten Trassen, das ist die HGÜ-Technik [...], die es bei uns in Deutschland nicht gibt, die es in Europa nicht gibt, sondern die mal in Nordamerika probiert wurde und in Afrika. Also wir haben überhaupt keine Erfahrung, ob damit gesundheitliche Beeinträchtigungen gegeben sind oder nicht. Und so lange das nicht geklärt ist, halten wir es für nicht vertret- 
bar, dass wir in Bayern oder überhaupt in Deutschland als Versuchskaninchen herhalten müssen."

Darüber hinaus werden Freileitungen, unabhängig von der Technik, tendenziell negativ bewertet. Abgesehen von einigen Teststrecken für Erdverkabelungen sahen die ursprünglichen Vorhabenplanungen Freileitungen vor. Letztere werden aber explizit mit ,gesundheitlichen Schäden“ (GT04) und „Angst vor Elektro-Smog“ (GT05) verbunden. Deren „biologische Wirkung“ seien „,bisher (ähnlich dem Mobilfunk) nicht eindeutig geklärt" (GT24). Freileitungen werden mit Risiken, Ängsten und Ungewissheiten - emotionale Bezugnahmen - verknüpft, denen Bürger ausgesetzt würden. Konkretisiert wird auf ein befürchtetes, erhöhtes Krebsrisiko, gerade ein Leukämie-Risiko bei Kindern, rekurriert - bisherige wissenschaftliche Ergebnisse bleiben allerdings hierzu uneindeutig (unter anderem SZ-06.02.2014, IP04 Amprion, IP13 Mediziner). Ob nun Gesundheitsrisiken als gegeben oder nicht gegeben bewertet werden, sie sind Bestandteil des Diskurses um den Stromnetzausbau, ohne dass eine Position die andere bisher in den Hintergrund drängen konnte.

Erdverkabelungen werden mit geringeren Belastungen assoziiert und somit eingefordert, insbesondere durch Bürgerinitiativen, wie sich medial (FO-30.05.2012) und innerhalb der norddeutschen Bürgerinitiativen (unter anderem IP-lok08 Bürgerinitiative „Keine 380 kV-Freileitung am Teuto“, IP-lok05 Kommunalvertreter Delligsen) zeigt. Vor diesem Hintergrund werden Erdkabel tendenziell als konfliktärmere Lösung und als anschlussfähiger an einen schnellen Netzausbau gerahmt (z. B. IP06 TenneT, IP11 DUH). Die Bundesnetzagentur fragt in diesem $\mathrm{Zu}-$ sammenhang, ob Erdkabel „den Planungsprozess beschleunigen und diese [...] eine Art Befriedungsfunktion“" erfüllen könnten - ,[w] as die Tendenz bezüglich der einzusetzenden Technik anbelangt, ist ein Großteil der Bevölkerung für die Erdverkabelung" - (IP03), womit Erdkabel und Akzeptanz argumentativ diskursiv verschnitten werden. Die politische Entscheidung vom Juli 2015, die großen Nord-Süd-Stromtrassen vorrangig mit Erdkabeln umzusetzen, kann in diese Richtung gedeutet werden.

\subsubsection{Sorge um wirtschaftliche und tourismusbezogene Einbußen}

Als weitere Argumente von Netzausbaugegnern und damit Momente im Stromnetzausbau-Diskurs fungieren Sorgen um sinkende Immobilienpreise und Einbußen im Tourismus. Die Bundesnetzagentur führt als Konfliktfelder „Wertverlust der Immobilien, de[n] geminderte[n] Erholungszweck und de[n] Wohnumfeldschutz" (IP03) an, Amprion spricht vom befürchteten „Wertverlust von Grundstücken und Häusern“ (IP05) und der Bund Naturschutz Bayern von „Grundstückswerte[n]“, die „,bedroht“" würden.
Für von Stromtrassen Betroffene ergebe sich ein möglicher Verlust von Grundstückswerten als Risiko, das vor den Planungen nicht ausreichend berücksichtigt worden sei. Die entsprechenden Befürchtungen finden sich innerhalb der Fallstudien auf lokaler Ebene wieder: „Wertverlust von Immobilien und Grundstücken sowie Einbußen für den Tourismus" (Mat-lok09 Webinhalt) werden im Fallkontext „Keine 380 kV-Freileitung am Teuto“ befürchtet, die „Vermeidung der Wertverluste bei Gebäuden und Grundstücken“ wird entsprechend angestrebt (Mat-lok10 Flyer der Bürgerinitiative). Gerade für den im Fallkontext interviewten Kommunalvertreter wird dieses Argument relevant, da er von verschiedenen Personen darauf angesprochen werde, dass Häuserverkäufe durch die Trassenplanungen erschwert oder nicht mehr möglich würden (IP-lok07). In Delligsen wird bemängelt, der „Wert der Immobilien“ werde „durch die Freileitungen erheblich vermindert“", ,das zunehmende touristische Interesse an der Region" würden Freileitungen „völlig zerstören“ (Mat-lok07 Webinhalt der Bürgerinitiative). Im Rahmen der Fallstudie „Pegnitz unter Strom" finden sich fast identische Formulierungen. Grundstückseigentümern drohe die „Entwertung ihrer Grundstücke, ihres Hab und Gutes“ (Mat-lok03 Schreiben an Bundesminister Gabriel), ,die Immobilien werden entwertet“, „Industriegebiet-Erweiterung, Gewerbegebietserweiterung werden zunichte gemacht, weil da keiner mehr baut" (IP-lok04 Bürgerinitiative).

Die bisherigen Planungen lassen nur Aussagen zu recht breiten Trassenkorridoren (500-1000 m Breite) zu. Wo die neuen Stromtrassen „genau“ entlang führen werden, ist erst noch festzulegen. ${ }^{4}$ Eine potenziell mögliche Betroffenheit führt allerdings zum jetzigen Zeitpunkt zu Sorgen, die schwer entkräftet werden können und diskursiv ausgehandelt werden.

\subsection{4 „Verschandelung “ und „Zerstörung “ von Natur und Landschaft}

Besonders häufig finden sich in den untersuchten Analysebestandteilen bei der Beleuchtung von Protestargumenten schließlich Bezugnahmen auf Natur und Landschaft. Im Rahmen medialer Darstellungen wird insbesondere auf (nicht vorhandene) Ästhetik - „schön“ versus „hässlich“ - von Freileitungen Bezug genommen: „Ob im Vereinigten Königreich, im Rest Europas oder hierzulande: Es wird nicht viele Menschen geben, die die stählernen Ungetüme besonders schön finden. Landschaftsliebhabern sind sie seit jeher ein Dorn im Auge" (SZ-12.06.2011). Natur, Landschaft und das Umfeld werden zu Momenten im Diskurs der Ablehnung von Freileitungen: „Natur- und Landschafts-

\footnotetext{
${ }_{4}$ http://www.netzausbau.de/5schritte/bundesfachplanung/de.html (28.06.2016).
} 
schutz sowie der Schutz des Wohnumfelds von Betroffenen sind dabei die wichtigsten Themen" (FO-07.10.2014). Deutlich umfänglicher werden die Bezugnahmen in den geführten Interviews mit Bundes- und Länderbezug: Der Netzausbau wird mit Veränderungen in den aktuellen materiellen Grundlagen von Landschaft in Verbindung gebracht. Zum zentralen Moment in diesem Diskursstrang wird die „Zerschneidung“ von Landschaft. Neue Infrastrukturen bewirkten die ,Zerschneidung der Landschaft" und gerade Masten hätten eine ,Zerschneidungswirkung“ (IP15 NABU, vergleichbar IP04 Amprion). In anderen Narrationen wird in Bezug auf die Sichtweise durch die bayerische Bevölkerung auf die „Verschandelung unserer Gegend“ und die „Leitung als optische Verschmutzung“ - ästhetisch argumentierend - rekurriert (IP16 Systemanalytiker). Landschaft wird konservativ-bewahrend mit Ästhetik (im Sinne eines ästhetischen Urteils „schön“) verschränkt (vgl. auch Leibenath/Otto 2012: 128; Kühne 2013a) und Stromtrassen werden aus diesem Deutungsmuster ausgeschlossen.

Aus Planungssicht sei es heute fast unmöglich, ,irgendwelche Leitungen so in die Landschaft zu bringen, dass man nicht irgendwie mit einem Schutzgut in Konflikt geraten würde, sei es der Naturschutz, sei es der Landschaftsschutz, sei es aber eben auch das Wohnumfeld“" (IP04). Auch wenn diese nach Einschätzung eines Mitarbeiters von Amprion ,in der Bevölkerung eigentlich weniger eine Rolle spielen“, seien „Naturschutz, Artenschutz und solche Dinge [...] rechtlich zwingend zu beachten“ und würden daher als Verhinderungsinstrumente angeführt (IP04). Landschaft, Natur und Schutz des Wohnumfeldes reihen sich regelmäBig aneinander, womit deren Verwobenheit ganz ,natürlich erscheint - in der Summe unterstreichen sie die Ablehnung von Freileitungsplanungen und erhalten so größere Relevanz.

Bürgerinitiativen (re)produzieren regelmäßig entsprechende Argumentationsmuster: Von 90 Bürgerinitiativen, die über einen Internet- oder Facebook-Auftritt verfügen, führen über $85 \%$ landschaftsbezogene und knapp $60 \%$ naturschutzfachliche Begründungen gegen bestehende Planungen an. Landschaft und Natur werden auch in den untersuchten Fallkontexten aufgegriffen - und dies ebenfalls sehr umfänglich. Dies zeigt sich bereits in den analysierten regionalen und lokalen Zeitungen, in denen beispielsweise kritisiert wird, dass ,mit der vorgesehenen Trassenführung eine erhebliche Zerstörung einer noch halbwegs intakten Landschaft" einhergehe (N-lok0525.02.2014) bzw. Landschaft und Natur ,brutal zerstört“ würden (N-lok04-14.11.2014, auch in N-lok04-21.06.2014 vergleichbar emotional gerahmt). In Bezug auf Landschaft wird im Fallkontext „Keine 380 kV-Freileitung am Teuto“ auf den „Erhalt der Landschaft am Teutoburger Wald durch Vermeidung ihrer Zerschneidung“ (Mat-lok09 Webinhalt) rekurriert. Für Vertreter der Bürgerinitiativen werden ent- sprechende Bezugnahmen zur Grundlage ihres Handelns „Wir wollen unsere Heimat und unsere Landschaft, unsere Natur erhalten“ (IP-lok08) - und damit gleichzeitig Ausgangspunkt, um Erdverkabelungen zu fördern. Auch in der zweiten norddeutschen Fallstudie „Delligsen in der Hilsmulde“ werden Natur- und Landschaftsschutz zu zentralen Aufgaben, die sich bereits im Vereinszweck der Bürgerinitiative widerspiegeln (Mat-lok06 Website der Kommune). Landschafts- und Naturzerstörungen werden bei den norddeutschen Fallstudien mit Freileitungen gekoppelt und zum Außen des Energiewende-Diskurses. An diesen werden hingegen Erdverkabelungen anschlussfähig, also Erdkabel zur Umsetzung der Energiewende.

Der Unterschied der norddeutschen zu den bayerischen Fallstudien liegt darin, dass in Bayern Bezugnahmen auf Landschaft und besonders auf „Heimat“ zu Begründungsmustern werden, die mit der grundlegenden Ablehnung der Gleichstrompassage Süd-Ost interferieren: Da die Gleichstrompassage Süd-Ost als insgesamt nicht erforderlich bewertet wird, werden „Landschafts- und Heimatzerstörung“ als ,sinnlos“ und ,völlig unnötig“ zurückgewiesen - und damit hegemonial verankert. Pegnitz stehe ,unter Strom. Das Damoklesschwert HGÜ Passage Süd-Ost schwebt über unseren Köpfen. Wir sind bedroht. Unsere Landschaft ist bedroht. [...]. Unsere Heimat ist bedroht" (Mat-lok03 Rede Bürgermeister). Eine Zustimmung zur Trasse wird vor diesem Hintergrund „moralisch verwerflich“. Auch die Wortwahl „Monsterstromtrasse“ (Mat-lok03 Pressemitteilung) zeugt von einer besonders ablehnenden Position und sehr emotionalen sowie ästhetischen Argumentationen. In Schnaittach heißt es unter anderem, die Trasse würde die Hälfte des Kommunalgebietes „optisch zerstör[en]“ bzw. „Wir kämpfen weiter gegen diese Monstertrasse, [...] denn diese Trasse zerstört unsere Heimat [...]. Die Leitung ist überflüssig, sie ist hässlich, sie ist monströs [...]. Dies werden wir nicht auf Kosten unserer Landschaft [...] dulden“ (Mat-lok02 Webinhalt der Bürgerinitiative). „Naturverlust“ (IP-lok01 Kommunalvertreter Schnaittach) wird zu einem weiteren Schlagwort, das mit Landschaftsschutz in Beziehung gesetzt wird. Gegenwärtige Zustände sollen geschützt werden, in die Masten nicht hineinpassen. Landschaft und Natur werden damit als fest und unumstößlich begriffen. Wandelbarkeit und Konstruktionscharakter werden ausgeblendet bzw. nicht wahrgenommen, womit jede Veränderung mit Nachdruck abgelehnt wird (vgl. ausführlich Kühne 2006; Kühne 2013b).

\section{Fazit: Verfestigungen spezifischer Positionen als Herausforderung für die Planung}

Welche Schlussfolgerungen lassen sich aus den dargestellten Ergebnissen ziehen? Die diskurstheoretische Perspekti- 
ve, die den Fokus auf die Veränderbarkeit sozialer Wirklichkeiten und gleichzeitig auf spezifische Verfestigungsprozesse sowie diskursive Brüche und Verschiebungen legt, ermöglicht es, den Blick darauf zu richten, welche Deutungsmuster sich derzeit zum Stromnetzausbau in Aushandlung befinden, sich in Teilen verfestigen und damit alternative Möglichkeiten in den Hintergrund drängen. Im Gegensatz zu bisherigen Untersuchungen zum Netzausbau ergibt sich damit ein spezifischer Fokus auf die (Re-)Produktion sozialer Wirklichkeiten. Es zeigt sich, dass in Teilen mehrere Diskursstränge bestehen, die sich überlagern und von der Grundausrichtung sogar teilweise eher ausschließen.

Wie dargestellt, wird gerade in den untersuchten Medien Google, SZ und Focus sowie in Interviews mit Ansprechpartnern der Bundes- und Länderebene der Stromnetzausbau und die weitere Umsetzung der Energiewende, gerade mit dem Ausbau erneuerbarer Energien und dem nach der Reaktorkatastrophe in Fukushima politisch (erneut) beschlossenen Ausstieg aus der Kernkraft bis 2022 verknüpft, miteinander verwoben und verankert. Abweichende Deutungsmuster, unter anderem in Bezug auf das Argument Kohlestromtransport, sind Teil des Diskurses, aber eher als Subdiskurse zu werten, die (bisher) keine hegemoniale Stellung im Verhältnis zur Positionierung von Bundesregierung, Bundesnetzagentur sowie Übertragungsnetzbetreibern und deren medialer (Re-)Produktion erlangen konnten.

Regional ergeben sich unterschiedliche Rahmungen der Energiewende, analytisch basierend auf der Auswertung der 90 ermittelten Bürgerinitiativen mit Internet/Facebook-Auftritt und fallstudienbezogen mit Schwerpunkt Nordrhein-Westfalen/Niedersachsen und Bayern beleuchtet. Bayerische Bürgerinitiativen, die sich konstituiert haben, sehen ihre dezentrale, regionale Energiewende gefährdet und halten neue Stromtrassen, explizit innerhalb der Fallstudien die Gleichstrompassage Süd-Ost, für nicht erforderlich. Massive Widerstände, die auch die Haltung der bayerischen Staatsregierung beeinflusst haben (vgl. in der Übersicht Abb. 4), zeugen davon und werden medial regional sowie lokal stark (re)produziert aufgeladen. Politisches Handeln wurde und wird als erforderlich angesehen - in Reaktion auf bürgerschaftliches Engagement, was dessen Macht und Durchsetzungspotential widerspiegelt. Dies zeigt auch die dilemmatische Position von Politik und ihrer Administration: Von ihnen wird ein hohes Durchsetzungsvermögen erwartet, allerdings nur dann, wenn es dazu bestimmt ist, die eigene Position durchzusetzen. Dieses wird gleichzeitig jedoch durch das Handeln von Bürgerinitiativen selbst unterminiert.

In Norddeutschland wird der Stromnetzausbau dagegen, ausgehend von den Positionierungen der Bürgerinitiativen in den untersuchten Internet-/Facebook-Profilen sowie der Fallstudien, als Konsequenz der Energiewende tendenziell akzeptiert. Befürworter sehen die Notwendigkeit, be- gründet über den Stromtransport von Nord nach Süd nur werden dort in Teilen intransparente Planungsprozesse oder "landschaftsverschandelnde“ und gesundheitsbedrohende Freileitungsplanungen kritisiert. Die vielfach geplanten Freileitungen werden dort eher zum Außen des Diskurses. Zur favorisierten Lösung werden Erdverkabelungen, wobei auch Freileitungen nach der Prüfung von Trassenalternativen oder Anpassungen bei den Trassenführungen in den Bereich des Möglichen rücken (vgl. auch Neukirch 2016).

Es ergeben sich entsprechend komplexe Aushandlungsprozesse - mit kognitiven, emotionalen und ästhetischen Bezugnahmen - um die Deutungsmacht, die Durchsetzungsfähigkeit spezifischer Positionen. Dies zeigt sich ganz grundsätzlich im Hinblick auf die Bewertung der Bedarfsfrage des Netzausbaus: Wird dieser akzeptiert oder dezidiert zurückgewiesen? Die Bejahung, unter anderem durch Bundesregierung, Übertragungsnetzbetreiber und Bundesnetzagentur, ist nicht so stark verankert, als dass, gerade regional und lokal wie zum Beispiel in Bayern, abweichende Haltungen nicht (große) Macht entfalten könnten. Wird die Äquivalenzbeziehung Energiewende-Netzausbau nicht grundsätzlich hinterfragt, ergeben sich wiederum vielfach Aushandlungsprozesse um Freileitungen versus Erdverkabelungen und die Prüfung von Trassenalternativen, verbunden mit Argumentationsmustern um die dargestellten Kritikpunkte wie Beteiligung, Technik, Gesundheit, Ökonomie, Natur und Landschaft - Muster, die regelmäßig und auf vergleichbare Weise vorgebracht werden und sich so diskursiv als „relevant“ und „,beachtenswert“ verfestigen. Aus praxisorientierter Perspektive (vgl. Chilla/Kühne/ Weber et al. 2015) sind es gerade ,extreme“ Positionen wie in Bayern, die Aushandlungsprozesse in Bezug auf Umsetzungen des Stromnetzausbaus herausfordern (vgl. Weber/ Kühne/Jenal et al. 2016). Wird der Bedarf grundlegend hinterfragt und jeder Umsetzungswille abgelehnt, werden konsensuale Lösungen (fast) unmöglich. Gleichzeitig besteht auf Seiten der zentralen Institutionen der NetzausbauBefürwortung die Notwendigkeit, den Netzausbaubedarf (noch) transparenter zu machen und zu veranschaulichen.

Werden dagegen bisherige Beteiligungsmöglichkeiten als unzureichend bewertet bzw. Alternativoptionen gewünscht, so kann über veränderte Beteiligungsprozesse und Debatten ein Lösungsansatz gefunden werden. Als schwer lösbares Problem erweist sich im Zuge des Stromnetzausbaus, dass der ermittelte Bedarf an neuen Stromtrassen sich nicht direkt in konkreten Trassenvarianten, sondern zunächst in recht vagen Korridoren niederschlägt. Auf diese Weise werden Unsicherheiten durch mögliche Betroffenheit, gefühlte Bedrohung des eigenen Lebensumfeldes, eher befördert. Noch umfänglichere Berücksichtigung von Sorgen, Befürchtungen und Ängsten, Informationsangebote und Dialogveranstaltungen erscheinen erforderlich, um 
innerhalb der Planfeststellungsverfahren nicht eine Flut von Einwendungen zu provozieren, die den konkreten Trassenbau zur never ending story werden lassen. Ob Erdkabel tatsächlich die Lösung sein können oder ob sich im nächsten Schritt auch Widerstand gegen Erdverkabelungen regen wird, bleibt abzuwarten.

Danksagung Das diesem Beitrag zu Grunde liegende Forschungsvorhaben wurde im Auftrag des Bundesamtes für Strahlenschutz (BfS) und des Bundesministeriums für Umwelt, Naturschutz, Bau und Reaktorsicherheit (BMUB) durchgeführt. Die Verantwortung für den Inhalt dieser Veröffentlichung liegt bei den Autoren. Wir danken dem Bundesamt und dem Bundesministerium für die Unterstützung. Unser besonderer Dank gilt auch unseren am Projekt beteiligten Kolleginnen und Kollegen Corinna Jenal, Kerstin Langer, Tina Sanio, Michael Igel und Tobias Sontheim sowie Cornelia Egblomassé-Roidl vom Bundesamt für Strahlenschutz.

\section{Literatur}

Agentur für Erneuerbare Energien (2015): Die deutsche Bevölkerung will mehr Erneuerbare Energien: Repräsentative Akzeptanzumfrage zeigt hohe Zustimmung für weiteren Ausbau. http://www. unendlich-viel-energie.de/die-deutsche-bevoelkerung-will-mehrerneuerbare-energien (13.06.2016).

Brailich, A.; Germes, M.; Schirmel, H.; Glasze, G.; Pütz, R. (2008): Die diskursive Konstitution von Großwohnsiedlungen in Deutschland, Frankreich und Polen. In: Europa Regional 16, 3, 113-128.

Bundesregierung (2015): Erneuerbare Energien. Ein neues Zeitalter hat begonnen. http://www.bundesregierung.de/Webs/Breg/DE/ Themen/Energiewende/EnergieErzeugen/ErneuerbareEnergienZeitalter/_node.html (13.06.2016).

Chilla, T.; Kühne, O.; Weber, F.; Weber, F. (2015): ,Neopragmatische“ Argumente zur Vereinbarkeit von konzeptioneller Diskussion und Praxis der Regionalentwicklung. In: Kühne, O.; Weber, F. (Hrsg.): Bausteine der Regionalentwicklung. Wiesbaden, 13-24.

Feneberg, B. (2014): Gegen die Stromtrasse: Widerstand über Grenzen hinweg. In: Augsburger Allgemeine vom 29.05.2014. http://www. augsburger-allgemeine.de/donauwoerth/Gegen-die-StromtrasseWiderstand-ueber-Grenzen-hinweg-id30003882.html (13.06.2016).

Gailing, L.; Leibenath, M. (2015): The Social Construction of Landscapes: Two Theoretical Lenses and their Empirical Applications. In: Landscape Research 40, 2, 123-138.

Glasze, G. (2007): Vorschläge zur Operationalisierung der Diskurstheorie von Laclau und Mouffe in einer Triangulation von lexikometrischen und interpretativen Methoden. In: Forum Qualitative Sozialforschung 8, 2, Artikel 14. http://www.qualitative-research. net/index.php/fqs/rt/printerFriendly/239/529 (13.06.2016).

Glasze, G. (2013): Politische Räume. Die diskursive Konstitution eines „geokulturellen Raums“ - die Frankophonie. Bielefeld.

Glasze, G.; Husseini, S.; Mose, J. (2009): Kodierende Verfahren in der Diskursforschung. In: Glasze, G.; Mattissek, A. (Hrsg.): Handbuch Diskurs und Raum. Theorien und Methoden für die Humangeographie sowie die sozial- und kulturwissenschaftliche Raumforschung. Bielefeld, 293-314.

Glasze, G.; Mattissek, A. (2009a): Die Hegemonie- und Diskurstheorie von Laclau und Mouffe. In: Glasze, G.; Mattissek, A. (Hrsg.): Handbuch Diskurs und Raum. Theorien und Methoden für die Humangeographie sowie die sozial- und kulturwissenschaftliche Raumforschung. Bielefeld, 153-179.

Glasze, G.; Mattissek, A. (Hrsg.) (2009b): Handbuch Diskurs und Raum. Theorien und Methoden für die Humangeographie sowie die sozial- und kulturwissenschaftliche Raumforschung. Bielefeld.
Glasze, G.; Weber, F. (2014): Die Stigmatisierung der banlieues in Frankreich seit den 1980er Jahren als Verräumlichung und Ethnisierung gesellschaftlicher Krisen. In: Europa Regional 20, 2012, $2-3,63-75$.

Gleitsmann, R.-J. (2011): Der Vision atomtechnischer Verheißungen gefolgt. Von der Euphorie zu ersten Protesten - die zivile Nutzung der Kernkraft in Deutschland seit den 1950er Jahren. In: Journal of New Frontiers in Spatial Concepts 3, 17-26.

Hübner, G.; Hahn, C. (2013): Akzeptanz des Stromnetzausbaus in Schleswig-Holstein. Abschlussbericht zum Forschungsprojekt. Halle. http://www.forum-netzintegration.de/uploads/media/ Akzeptanz_Stromnetz_SH_Mai2013_web.pdf (13.06.2016).

Jarass, L.; Obermair, G. M. (2012): Welchen Netzumbau erfordert die Energiewende? Münster.

Koch, S.; Odparlik, L.; Köppel, J. (2014): Wo steht die Partizipation beim Netzausbau? Eine Analyse der Beteiligungsverfahren zu ausgewählten Projekten aus dem Bedarfsplan des Energieleitungsausbaugesetzes. In: Naturschutz und Landschaftsplanung $46,4,116-123$.

Kühne, O. (2006): Landschaft in der Postmoderne. Das Beispiel des Saarlandes. Wiesbaden.

Kühne, O. (2012): Stadt - Landschaft - Hybridität. Ästhetische Bezüge im postmodernen Los Angeles mit seinen modernen Persistenzen. Wiesbaden.

Kühne, O. (2013a): Landschaftsästhetik und regenerative Energien Grundüberlegungen zu De- und Re-Sensualisierungen und inversen Landschaften. In: Gailing, L.; Leibenath, M. (Hrsg.): Neue Energielandschaften - Neue Perspektiven der Landschaftsforschung. Wiesbaden, 101-120.

Kühne, O. (2013b): Landschaftstheorie und Landschaftspraxis. Eine Einführung aus sozialkonstruktivistischer Perspektive. Wiesbaden.

Laclau, E. (1993): Discourse. In: Goodin, R. E.; Pettit, P. (Hrsg.): A companion to contemporary political philosophy. Oxford, 431-437.

Laclau, E. (1994): Introduction. In: Laclau, E. (Hrsg.): The Making of Political Identities. London, 1-8.

Laclau, E. (2007): On Populist Reason. London, New York.

Laclau, E.; Mouffe, C. (1985): Hegemony and socialist strategy. Towards a radical democratic politics. London.

Landkreis Hameln-Pyrmont (2014): 17 Bündnispartner vom hohen Norden bis nach Bayern verständigen sich auf die Hamelner Erklärung! Hameln-Pyrmont, 12.12.2014. http://www. hamelner-erklaerung.de/_pdf/Hamelner_Erklaerung_25022015. pdf (13.06.2016).

Leibenath, M. (2014): Landschaft im Diskurs: Welche Landschaft? Welcher Diskurs? Praktische Implikationen eines alternativen Entwurfs konstruktivistischer Landschaftsforschung. In: Naturschutz und Landschaftsplanung 46, 4, 124-129.

Leibenath, M.; Otto, A. (2012): Diskursive Konstituierung von Kulturlandschaft am Beispiel politischer Windenergiediskurse in Deutschland. In: Raumforschung und Raumordnung 70, 2, $119-131$.

Leibenath, M.; Otto, A. (2013): Windräder in Wolfhagen - eine Fallstudie zur diskursiven Konstituierung von Landschaften. In: Leibenath, M.; Heiland, S.; Kilper, H.; Tzschaschel, S. (Hrsg.): Wie werden Landschaften gemacht? Sozialwissenschaftliche Perspektiven auf die Konstituierung von Kulturlandschaften. Bielefeld, 205-236.

Marg, S.; Geiges, L.; Butzlaff, F.; Walter, F. (Hrsg.) (2013): Die neue Macht der Bürger. Was motiviert die Protestbewegungen? BP-Gesellschaftsstudie. Reinbek bei Hamburg.

Mattissek, A. (2005): Diskursive Konstitution von Sicherheit im öffentlichen Raum am Beispiel Frankfurt am Main. In: Glasze, G.; Pütz, R.; Rolfes, M. (Hrsg.): Diskurs, Stadt, Kriminalität. Städtische (Un-)Sicherheiten aus der Perspektive von Stadtforschung und kritischer Kriminalgeographie. Bielefeld, 105-136. 
Mattissek, A. (2008): Die neoliberale Stadt. Diskursive Repräsentationen im Stadtmarketing deutscher Großstädte. Bielefeld.

Neukirch, M. (2014): Konflikte um den Ausbau der Stromnetze. Status und Entwicklung heterogener Protestkonstellationen. Stuttgart. = SOI Discussion Paper 2014-01. http://www.uni-stuttgart.de/ soz/oi/publikationen/soi_2014_1_Neukirch_Konflikte_um_den_ Ausbau_der_Stromnetze.pdf (13.06.2016).

Neukirch, M. (2015): Mehr Netzausbau mit weniger Kohle? Zwei ökologische Perspektiven auf Korridor D. In: Zeitschrift für Politische Ökologie 141, 132-135.

Neukirch, M. (2016): Protests against German electricity grid extension as a new social movement? A journey into the areas of conflict. In: Energy, Sustainability and Society. doi 10.1186/s13705-0160069-9.

Nonhoff, M. (2006): Politischer Diskurs und Hegemonie. Das Projekt „Soziale Marktwirtschaft“. Bielefeld.

Pan, B.; Hembrooke, H.; Joachims, T.; Lorigo, L.; Gay, G.; Granka, L. (2007): In Google We Trust: Users' Decisions on Rank, Position, and Relevance. In: Journal of Computer-Mediated Communication $12,3,801-823$.

Phillips, L.; Jørgensen, M. W. (2002): Discourse Analysis as Theory and Method. London.

Renn, O. (2005): Technikakzeptanz: Lehren und Rückschlüsse der Akzeptanzforschung für die Bewältigung des technischen Wandels. In: Zeitschrift des ITAS zur Technikfolgenabschätzung 14, 3, 29-38.

Riegel, C.; Brandt, T. (2015): Eile mit Weile - Aktuelle Entwicklungen beim Netzausbau. In: ARL-Nachrichten 45, 2, 10-16.

Schirmel, H. (2011): Sedimentierte Unsicherheitsdiskurse. Die diskursive Konstitution von Berliner Großwohnsiedlungen als unsichere Orte und Ziel von Sicherheitspolitiken. Erlangen. = Erlanger Geographische Arbeiten 39.

Schmidt, G. (2015): Energiewende ohne Regionalplanung? Prof. Dr. Axel Priebs und Dr. Holger Schmitz im Gespräch über die aktuelle Rechtslage bei der Gestaltung der Energiewende und die Rolle der Landes- und Regionalplanung. In: ARL-Nachrichten 45, 2, $17-21$.

Schmidt, J.-H. (2011): Das neue Netz. Merkmale, Praktiken und Folgen des Web 2.0. Konstanz.

Schweizer-Ries, P. (2010): Umweltpsychologische Untersuchung der Akzeptanz von Maßnahmen zur Netzintegration Erneuerbarer Energien in der Region Wahle-Mecklar (Niedersachsen und Hessen). Magdeburg. http://www.fg-umwelt.de/assets/ files/Akzeptanz\%20Netzausbau/Abschlussbericht_Akzeptanz_ Netzausbau_Juni2010.pdf (13.06.2016).

Somers, M. (1994): The narrative constitution of identity: A relational and network approach. In: Theory and Society 23, 5, 605-649.
Stegert, P.; Klagge, B. (2015): Akzeptanzsteigerung durch Bürgerbeteiligung beim Übertragungsnetzausbau? Theoretische Überlegungen und empirische Befunde. In: Geographische Zeitschrift 103, 3, 171-190.

Thiem, N.; Weber, F. (2011): Von eindeutigen Uneindeutigkeiten Grenzüberschreitungen zwischen Geografie und Literaturwissenschaft im Hinblick auf Raum und Kartografie. In: Gubo, M.; Kypta, M.; Öchsner, F. (Hrsg.): Kritische Perspektiven: „Turns“, Trends und Theorien. Berlin, 171-193.

Torfing, J. (1999): New theories of discourse: Laclau, Mouffe and Žižek. Oxford.

Übertragungsnetzbetreiber (2014a): Netzentwicklungsplan Strom 2014. Zweiter Entwurf. Teil 1. Berlin. http://www. netzentwicklungsplan.de/_NEP_file_transfer/NEP_2014_2 Entwurf_Teil1.pdf (29.04.2016).

Übertragungsnetzbetreiber (2014b): Netzentwicklungsplan Strom 2014. Zweiter Entwurf. Teil 2. Berlin. http://www. netzentwicklungsplan.de/_NEP_file_transfer/NEP_2014_2_ Entwurf Teil2.pdf (29.04.2016)

Weber, F. (2013): Soziale Stadt - Politique de la Ville - Politische Logiken. (Re-)Produktion kultureller Differenzierungen in quartiersbezogenen Stadtpolitiken in Deutschland und Frankreich. Wiesbaden.

Weber, F. (2015): Diskurs - Macht - Landschaft. Potenziale der Diskurs- und Hegemonietheorie von Ernesto Laclau und Chantal Mouffe für die Landschaftsforschung. In: Kost, S.; Schönwald, A. (Hrsg.): Landschaftswandel - Wandel von Machtstrukturen. Wiesbaden, 97-112.

Weber, F.; Jenal, C.; Kühne, O. (2016): Der Stromnetzausbau als konfliktträchtiges Terrain. In: Umwelt und Mensch Informationsdienst $1,50-56$.

Weber, F.; Kühne, O.; Jenal, C.; Sanio, T.; Langer, K.; Igel, M. (2016): Analyse des öffentlichen Diskurses zu gesundheitlichen Auswirkungen von Hochspannungsleitungen - Handlungsempfehlungen für die strahlenschutzbezogene Kommunikation beim Stromnetzausbau. Ressortforschungsberichte zur kerntechnischen $\mathrm{Si}$ cherheit und zum Strahlenschutz. Freising. https://doris.bfs.de/ jspui/bitstream/urn:nbn:de:0221-2016050414038/3/BfS_2016_ 3614S80008.pdf (04.05.2016).

Zimmer, R.; Kloke, S.; Gaedtke, M. (2012): Der Streit um die Uckermarkleitung - Eine Diskursanalyse. Studie im Rahmen des UfU-Schwerpunktes „Erneuerbare Energien im Konflikt“. Berlin. = UfU-Paper 3/12. http://digital.zlb.de/viewer/content? action=application\&sourcepath=15702859/Streit_um_die_ Uckermarkleitung.pdf\&format=pdf (13.06.2016). 